\title{
A Mathematical Approach to the Effective Hamiltonian in Perturbed Periodic Problems
}

\author{
C. Gerard ${ }^{1}$, A. Martinez ${ }^{2}$ and J. Sjöstrand ${ }^{3}$ \\ ${ }^{1}$ Centre de Mathématiques, Ecole Polytechnique, F-91128 Palaiseau Cedex, France \\ 2 Université de Paris-Nord, Département de Mathématiques, Av. J.B. Clément, F-93430 \\ Villetaneuse, France \\ 3 Université de Paris-Sud, Département de Mathématiques, F-91405 Orsay Cedex, France
}

Received April 5, 1990; in revised form April 1, 1991

\begin{abstract}
We describe a rigorous mathematical reduction of the spectral study for a class of periodic problems with perturbations which gives a justification of the method of effective Hamiltonians in solid state physics. We study the partial differential operators of the form $P=P\left(h y, y, D_{y}+A(h y)\right.$ ) on $\mathbb{R}^{n}$ (when $h>0$ is small enough), where $P(x, y, \eta)$ is elliptic, periodic in $y$ with respect to some lattice $\Gamma$, and admits smooth bounded coefficients in $(x, y) . A(x)$ is a magnetic potential with bounded derivatives. We show that the spectral study of $P$ near any fixed energy level can be reduced to the study of a finite system of $h$-pseudodifferential operators $\mathscr{E}\left(x, h D_{x}, h\right)$, acting on some Hilbert space depending on $\Gamma$. We then apply it to the study of the Schrödinger operator when the electric potential is periodic, and to some quasiperiodic potentials with vanishing magnetic field.
\end{abstract}

\section{Introduction}

The purpose of this paper is to give a rigorous mathematical treatment of an approximation widely used in solid state physics, namely the method of the effective Hamiltonian.

Let us briefly describe the essential ideas of this method: a typical problem to which this approximation is applied is the motion of an electron in a periodic crystal with a small external magnetic field. This problem is described by the following Hamiltonian:

$$
H=\sum_{1}^{3}\left(D_{y_{j}}+A_{j}(h y)\right)^{2}+V(y),
$$

where $V$ is a real potential, $\Gamma$-periodic for a lattice $\Gamma$ in $\mathbb{R}^{3}$ describing the periodic crystal, and $A(x)$ is a function from $\mathbb{R}^{3}$ into $\mathbb{R}^{3 *}$ (in other words a 1 -form), which 
is the vector potential of the magnetic field $B=d A$. We assume that all the derivatives (of order $\geqq 1$ ) of $A$ are bounded functions.

The effective Hamiltonian approximation is to replace, for $h$ small, $H$ by the collection of $h$-pseudodifferential operators:

$$
E_{j}\left(h D_{x}+A(x)\right) \quad \text { for } \quad j \in \mathbb{N},
$$

where $E_{j}(\theta)$ are the Bloch eigenvalues of $D_{y}^{2}+V(y)$.

In solid state physics, one then usually uses W.K.B. approximations to study the spectrum of $E_{j}\left(h D_{x}+A(x)\right)$. In the case of constant magnetic fields (i.e. when $A_{j}$ are linear) rigorous reductions from (0.1) to $(0.2)$ have been given by Nenciu [Ne] (single band case) and by Helffer-Sjöstrand [He-Sj1]. These reductions use Wannier functions.

We refer to the article of Guillot-Ralston-Trubowitz $[\mathrm{Gu}-\mathrm{Ra}-\mathrm{Tr}]$ and the survey by Buslaev $[\mathrm{Bu}]$, where the problem of constructing W.K.B. solutions using an effective Hamiltonian is studied in detail. An extensive bibliography on the physics literature about the effective Hamiltonian method can also be found in [Bu].

Our goal in this paper is to give a rigorous way to recover the spectrum of $H$ near some energy level $\lambda_{0}$ (and possibly also the nature of the spectrum) by studying systems of $h$-pseudodifferential operators which have a principal symbol quite close to $E_{j}(\xi+A(x))-\lambda$, where $\lambda$ is the spectral parameter.

Using our results we can for example justify the use of the effective Hamiltonian approximation for $(0.1)$ modulo error terms of order $\mathcal{O}(h)$ near a simple band of the spectrum of $D_{y}^{2}+V(y)$.

Let us now describe our results more in detail:

We consider partial differential operators of the form $P_{0}=P\left(h y, y, D_{y}+A(h y)\right)$, where $P(x, y, \eta)$ is an elliptic polynomial in $\eta, \Gamma$-periodic in $y$ for some lattice $\Gamma$, and with smooth bounded coefficients in $(x, y) . A(y)$ is a vector potential with all its non zero derivatives bounded. (See Sect. I for the precise hypotheses.)

The observation of Buslaev [Bu] (implicitly also by Guillot-Ralston-Trubowitz [Gu-Ra-Tr]), which is directly related to the two-scale expansion method is the following one: if $u(x, y) \in D^{\prime}\left(\mathbb{R}_{x}^{n} \times \mathbb{R}_{y}^{n}\right)$ is a solution $\Gamma$-periodic in $y$ of:

$$
P\left(x, y, h D_{x}+D_{y}+A(x)\right) u=\lambda u
$$

then $\tilde{u}=u(h y, y)$ satisfies:

$$
P_{0} \tilde{u}=\lambda \tilde{u} .
$$

Buslaev then uses this idea to construct asymptotic solutions of (0.4) by considering $P\left(x, y, h D_{x}+D_{y}+A(x)\right)$ as a $h$-pseudodifferential operator in $x$ with operator valued symbol. This in turn is related to the study of operators $(0.2)$. While this procedure gives asymptotic solutions of (0.4), it is not clear how to relate the spectrum of $P_{0}$ near an energy level $\lambda_{0}$ to that of $P=P\left(x, y, h D_{x}+D_{y}+A(x)\right)$.

We give a complete answer to this question in two cases. We obtain a $N \times N$ system of $h$-pseudodifferential operators of order $0, E_{-+}\left(x, h D_{x}+A(x), \lambda, h\right)$, (which will be our effective Hamiltonian) such that the symbol $E_{-+}(x, \xi, \lambda, h)$ is $\Gamma^{*}$-periodic in $\xi$ where $\Gamma^{*}$ is the dual lattice of $\Gamma$ (note that this property is shared by the $E_{j}(\xi)$ in $\left.(0.2)\right)$ and such that one has the following equivalence:

For $h$ small enough, $\lambda$ is in the spectrum of $P_{0}$ (with its natural domain, see 
Sect. I) if and only if 0 is in the spectrum of $E_{-+}\left(x, h D_{x}+A(x), \lambda, h\right)$ acting on the Hilbert space $\left(V_{0}\right)^{N}$, where

$$
V_{0}=\left\{u=\sum_{\gamma \in \Gamma} c_{\gamma} \delta(x-h \gamma),\left(c_{\gamma}\right) \in l^{2}(\Gamma)\right\} .
$$

(See Theorem 3.7 and Corollary 3.8.)

In the case when all the operators $P_{z}=P\left(z+h y, y, D_{y}+A(z+h y)\right)$ are isospectral to $P_{0}$ then the same result holds if we consider now $E_{-+}\left(x, h D_{x}+A(x), \lambda, h\right)$ acting simply on $L^{2}\left(\mathbb{R}_{x}^{n}, \mathbb{C}^{N}\right)$. (See Remark 1.2 and Theorem 2.4.) This is for example the case for (0.1) when $A$ is linear. We then apply these general results to Schrödinger operators. We consider periodic Schrödinger operators with magnetic fields and also some quasiperiodic Schrödinger operators.

For instance we construct a quasiperiodic Schrödinger operator in one dimension which yields an effective Hamiltonian arbitrarily close to Harper's operator $\cos h D_{x}+\cos x$.

Let us now give the plan of the paper.

In Sect. I, a general reduction scheme is introduced. This reduces the spectral study of $P_{0}$ to that of $P$ acting on a suitable Hilbert space.

In Sect. II, a "Grushin problem" is constructed to study the spectrum of $P$ on $L^{2}\left(\mathbb{R}_{x}^{n}, L^{2}\left(\mathbb{R}_{y}^{n} / \Gamma\right)\right)$. This is done by considering $P$ as an $h$-pseudodifferential operator in $x$ with operator valued symbol. With the help of this Grushin problem, we prove Theorem 2.4 .

In Sect. III, the methods of Sect. II are applied directly to the spectrum of $P_{0}$ to prove the above mentioned spectral equivalence.

Finally some examples are discussed in Sect. IV, including periodic and some quasiperiodic Schrödinger operators with magnetic fields.

Some technical results on magnetic Sobolev spaces and on pseudodifferential calculus with operator valued symbols are given in an Appendix.

\section{A General Reduction Scheme}

In this section we introduce a method inspired by a work of Buslaev [Bu] to study the spectrum of partial differential operators of the type (0.1).

We consider a function $P(x, y, \eta) \in C^{\infty}\left(\mathbb{R}^{3 n}\right)$ which is real valued and satisfies the following properties:

$P$ is a polynomial of degree $m$ with respect to $\eta$,

$$
\left\{\begin{array}{l}
P(x, y+\gamma, \eta)=P(x, y, \eta) \quad \forall \gamma \in \Gamma, \\
\text { where } \Gamma \text { is a lattice } \bigoplus_{i=1}^{n} \mathbb{Z} e_{i} \\
\text { for a basis }\left(e_{1}, \ldots, e_{n}\right) \text { of } \mathbb{R}^{n}
\end{array}\right.
$$

If

$$
P(x, y, \eta)=\sum_{|\alpha| \leqq m} a_{\alpha}(x, y) \eta^{\alpha} \quad \text { and } \quad p_{j}(x, y, \eta)=\sum_{|\alpha|=j} a_{\alpha}(x, y) \eta^{\alpha}
$$


then

$$
\begin{gathered}
\left|\partial_{x}^{\beta} \partial_{y}^{\gamma} a_{\alpha}(x, y)\right| \leqq C_{\beta, \gamma} \quad \forall \alpha, \beta, \gamma \in \mathbb{N}^{n} \\
p(x, y, \eta) \stackrel{\text { def }}{=} p_{m}(x, y, \eta) \quad \text { satisfies: } \\
p(x, y, \eta) \geqq \frac{1}{C_{0}}|\eta|^{m} \quad \text { for some } \quad C_{0}>0 .
\end{gathered}
$$

We shall also admit a magnetic field and the corresponding vector potential will be given by $A=A(x) \in C^{\infty}\left(\mathbb{R}^{n}, \mathbb{R}^{n *}\right)$.

We assume that

$$
\forall \alpha \in \mathbb{N}^{n} \backslash\{0\}, \quad \exists C_{\alpha} \text { such that }\left|\partial_{x}^{\alpha} A\right| \leqq C_{\alpha} .
$$

Before going on, let us say a word about the quantization we use in this work. We will always use the standard Weyl quantization of symbols: if $P(x, \eta)$ is a function on $T^{*} \mathbb{R}^{n}$ satisfying suitable estimates, $P^{w}\left(y, D_{y}\right)$ is the operator defined by:

$$
P^{w}\left(y, D_{y}\right) u(y)=(2 \pi)^{-n} \int e^{i\left(y-y^{\prime}\right) \cdot \eta} P\left(\frac{y+y^{\prime}}{2}, \eta\right) u\left(y^{\prime}\right) d y^{\prime} d \eta
$$

for $u \in S\left(\mathbb{R}^{n}\right)$.

Sometimes we will quantize a function $P(x, y, \xi, \eta)$ only with respect to the variables $(y, \eta)$ : in this case we will denote by $P^{w}\left(x, y, \xi, D_{y}\right)$ the operator obtained as above by considering $(x, \xi)$ as parameters.

Finally when $P(x, \xi)$ is a function on $T^{*} \mathbb{R}^{n}$ (possibly operator valued, see Appendix B), we denote by $P^{w}\left(x, h D_{x}\right)$ the semiclassical quantization obtained as above by quantizing $P(x, h \xi)$.

We start by considering the operator $P=P^{w}\left(x, y, h D_{x}+D_{y}+A(x)\right)$, which is the quantization of $P(x, y, h \xi+\eta+A(x))$. We will see later in this section that the operator $P_{0}=P^{w}\left(h y, y, D_{y}+A(h y)\right)$ can be viewed as the restriction of $P$ to the linear subspace $x=h y$.

To study $P$ we make the change of variables:

$$
(x, y) \mapsto(\tilde{x}, \tilde{y})=(x-h y, y) .
$$

Then using the invariance of Weyl's quantization by metaplectic transformations (see [Hö]) we see, by conjugating $P$ by the change of variables (1.2), that $P$ is transformed into:

$$
\tilde{P}=P^{w}\left(\tilde{x}+h \tilde{y}, \tilde{y}, D_{\tilde{y}}+A(\tilde{x}+h \tilde{y})\right) .
$$

We will see in Appendix A that $\tilde{P}$ is essentially self adjoint on $C_{0}^{\infty}\left(\mathbb{R}^{2 n}\right)$ and self adjoint with domain

$$
\widetilde{\mathscr{E}}_{m}=\left\{u \in L^{2}\left(\mathbb{R}^{2 n}\right)\left|\left(D_{\tilde{y}}+A(\tilde{x}+h \tilde{y})\right)^{\alpha} u \in L^{2}\left(\mathbb{R}^{2 n}\right), \forall\right| \alpha \mid \leqq m\right\} .
$$

Using the change of variables (1.2) (which can be realized as a unitary transformation) we get that $P$ is essentially self adjoint on $C_{0}^{\infty}\left(\mathbb{R}^{2 n}\right)$ and self adjoint with domain:

$$
\mathscr{E}_{m}=\left\{u \in L^{2}\left(\mathbb{R}^{2 n}\right)\left|\left(D_{y}+h D_{x}+A(x)\right)^{\alpha} u \in L^{2}\left(\mathbb{R}^{2 n}\right), \forall\right| \alpha \mid \leqq m\right\} .
$$


To study $P$ we will use the Floquet-Bloch reduction in the $y$ variable (see for example $[\mathrm{Sk}])$.

For $\theta \in \mathbb{R}^{n *} / \Gamma^{*}$, where $\Gamma^{*}$ is the dual lattice of $\Gamma$ consisting of all $\gamma^{*} \in \mathbb{R}^{n *}$ such that $\gamma^{*} \cdot \gamma \in 2 \pi \mathbb{Z}$ for every $\gamma \in \Gamma$, we put:

$$
\mathscr{U} u(x, y, \theta)=\sum_{\gamma \in \Gamma} e^{i \gamma \cdot \theta} u(x, y-\gamma)
$$

$\mathscr{U}$ is unitary from $L^{2}\left(\mathbb{R}_{x, y}^{2 n}\right)$ into

$$
\begin{aligned}
& \mathscr{F}_{0}=\frac{\overline{\text { def }}}{=}\left\{v(x, y, \theta) \in L_{\text {loc }}^{2}\left(\mathbb{R}^{3 n}\right) \mid v(x, y+\gamma, \theta)=e^{i \gamma \cdot \theta} v(x, y, \theta),\right. \\
&\left.v\left(x, y, \theta+\gamma^{*}\right)=v(x, y, \theta) \text { and }|v(\cdot, \cdot \theta)| \in L^{2}\left(\mathbb{R}^{n *} / \Gamma^{*}, L^{2}\left(\mathbb{R}_{x}^{n} \times \mathbb{R}_{y}^{n} / \Gamma\right)\right)\right\}
\end{aligned}
$$

when $\mathscr{F}_{0}$ is equipped with its natural scalar product.

We see that $\mathscr{U}$ commutes formally with $\left(h D_{x}+D_{y}+A(x)\right)^{\alpha}$, so for every $k \in \mathbb{N}, \mathscr{U}$ is unitary from $\mathscr{E}_{k}$ into

$$
\mathscr{F}_{k}=\left\{v \in \mathscr{F}_{0}\left|\left(h D_{x}+D_{y}+A(x)\right)^{\alpha} v \in \mathscr{F}_{0}, \forall\right| \alpha \mid \leqq k\right\}
$$

In particular $P$ with domain $\mathscr{E}_{m}$ is unitarily equivalent to the same formal differential operator $P$ acting on $\mathscr{F}_{0}$ with domain $\mathscr{F}_{m}$.

In order to eliminate the $\theta$-dependence as much as possible, we now consider the operator:

$$
\tilde{\mathscr{U}}: u \mapsto e^{i x \cdot \theta / h-i y \cdot \theta} \mathscr{U} u(x, y, \theta) .
$$

Then it is straightforward to check the following facts:

$$
\left\{\begin{array}{l}
\tilde{\mathscr{U}} \text { is unitary from } L^{2}\left(\mathbb{R}^{2 n}\right) \text { into } \\
\mathscr{H}_{0}=\left\{v \in L_{\text {loc }}^{2}\left(\mathbb{R}^{3 n}\right) \mid v(x, y+\gamma, \theta)=v(x, y, \theta),\right. \\
v\left(x, y, \theta+\gamma^{*}\right)=e^{i(x / h-y) \gamma^{*}} v(x, y, \theta), \\
\left.\int_{\mathbb{R}^{n} \times \mathbb{R}^{n} / \Gamma \times \mathbb{R}^{n * / \Gamma^{*}}}|v(x, y, \theta)|^{2} d x d y d \theta<+\infty\right\} .
\end{array}\right.
$$

$\tilde{\mathscr{U}}$ sends $\mathscr{E}_{m}$ into $\mathscr{H}_{m}=\left\{v \in \mathscr{H}_{0}\left|\left(h D_{x}+D_{y}+A(x)\right)^{\alpha} v \in \mathscr{H}_{0},\right| \alpha \mid \leqq m\right\}$.

$\left\{\begin{array}{l}\tilde{\mathscr{U}} P \tilde{\mathscr{U}}^{-1}=P, \text { where } P \text { in the right-hand side is the differential operator } \\ P^{w}\left(x, y, h D_{x}+D_{y}+A(x)\right) \text { acting on } \mathscr{H}_{0} \text { with domain } \mathscr{H}_{m} .\end{array}\right.$

We can also write the operator $P$ in the right-hand side of (1.6) as:

$$
P=\int_{E^{*}}^{\oplus} P d \theta
$$

where we consider $\mathscr{H}_{0}$ as the space of measurable functions $v(\theta)$ with values in the space

$$
\mathscr{K}_{0}=\left\{\left.u \in L_{\mathrm{loc}}^{2}\left(\mathbb{R}_{x, y}^{2 n}\right)\left|u(x, y+\gamma)=u(x, y), \int_{\mathbb{R}_{x}^{n} \times \mathbb{R}_{y}^{n} / \Gamma}\right| u(x, y)\right|^{2} d x d y<\infty\right\}
$$


such that: $v\left(\cdot, ; \theta+\gamma^{*}\right)=e^{i(x / h-y) \cdot \gamma^{*}} v(\cdot, \cdot, \theta)$ and:

$$
\int_{\mathbb{R}^{n^{*} / \Gamma^{*}}}\|v(\theta)\|_{\mathscr{K}_{0}}^{2} d \theta<+\infty
$$

and where $E^{*} \subset \mathbb{R}^{n^{*}}$ is a fundamental domain for $\Gamma^{*}$.

Accordingly $P$ in the right-hand side of (1.7) is the differential operator $P=P^{w}\left(x, y, h D_{x}+D_{y}+A(x)\right)$ acting in $\mathscr{K}_{0}$ which is selfadjoint with domain

$$
\mathscr{K}_{m}=\left\{u \in \mathscr{K}_{0}\left|\left(h D_{x}+D_{y}+A(x)\right)^{\alpha} u \in \mathscr{K}_{0}, \forall\right| \alpha \mid \leqq m\right\} .
$$

Since the spectrum of $P$ in the right-hand side of (1.7) is $\theta$-independent, we have proven the following result:

Proposition 1.1. The spectrum of $P$ acting on $L^{2}\left(\mathbb{R}^{2 n}\right)$ with domain $\mathscr{E}_{m}$ is the same as the spectrum of $P$ acting on $\mathscr{K}_{0}$ with domain $\mathscr{K}_{m}$.

(Remark that the nature of the spectrum is not necessarily the same for the two operators.)

Remark 1.2. Let us now indicate how one can use Proposition 1.1 to study the spectrum of $P_{0}$ in some cases.

Assume that $P(x, y, \eta)$ is such that the spectrum of $P_{z}=P^{w}\left(z+h y, y, D_{y}+A(z+h)\right)$ acting on $L^{2}\left(\mathbb{R}_{y}^{n}\right)$ with domain

$$
H_{m, A}=\left\{u \in L^{2}\left(\mathbb{R}_{y}^{n},\left|\left(D_{y}+A(h y)\right)^{\alpha} u \in L^{2}\left(\mathbb{R}_{y}^{n}\right), \alpha \in \mathbb{N}^{n},\right| \alpha \mid \leqq m\right\}\right.
$$

is independent of $z$. ( $P_{z}$ is selfadjoint in $H_{m, A}$ and essentially selfadjoint on $C_{0}^{\infty}\left(\mathbb{R}_{y}^{n}\right)$ by the arguments of Appendix A.)

This happens when $P_{0}$ is the Hamiltonian of a particle in a constant magnetic field with a periodic potential or with some class of quasiperiodic potentials. (See Sect. IV.)

Then it is straightforward to justify the following formal identity:

$$
\tilde{P}=\int_{\mathbb{R}^{n}}^{\oplus} P_{z} d z
$$

In fact (see [Re-Si]) we just have to check that $z \mapsto\left(P_{z}+i\right)^{-1}$ is weakly measurable, which follows easily from the second resolvent formula and hypotheses (H.3), (H.5).

Then we get: $\sigma(P)=\sigma(\widetilde{P})=\bigcup_{z \in \mathbb{R}^{n}} \sigma\left(P_{z}\right)=\sigma\left(P_{0}\right)$. So in this case $P_{0}$ has the same spectrum as $P$ acting on $\mathscr{K}_{0}$ with domain $\mathscr{K}_{m}$.

In Sect. II, we will study $P$ with domain $K_{m}$ by considering it as an operator valued $h$-pseudodifferential operator in $x$ with symbol $P^{w}\left(x, y, \xi+D_{y}+A(x)\right)$. $x=h y$.

We now consider the operator $P_{0}$ as a restriction of $P$ to the linear subspace

Let us consider $P_{0}=P^{w}\left(h y, y, D_{y}+A(h y)\right)$ with domain $H_{m, A}$.

In Appendix $\mathrm{A}$, we show that $P_{0}$ is essentially selfadjoint on $C_{0}^{\infty}\left(\mathbb{R}^{n}\right)$ and selfadjoint on $H_{m, A}$.

Using the change of variables (1.1) it is easy to see that $P_{0}$ acting on $L^{2}\left(\mathbb{R}^{n}\right)$ with domain $H_{m, A}$ is unitarily equivalent to $P$ acting on the Hilbert space:

$$
\left\{u(y) \delta(x-h y), u \in L^{2}\left(\mathbb{R}_{y}^{2}\right)\right\},
$$


with domain:

$$
\left\{u(y) \delta(x-h y), u \in H_{m, A}\right\} .
$$

(The norm of $u(y) \delta(x-h y)$ is the norm of $u$ in the corresponding space.)

This follows from the fact that

$$
P^{w}\left(\tilde{x}+h \tilde{y}, \tilde{y}, D_{\tilde{y}}+A(\tilde{x}+h \tilde{y})\right)(\delta(\tilde{x}) \otimes u(\tilde{y}))=\delta(\tilde{x}) \otimes P^{w}\left(h \tilde{y}, \tilde{y}, D_{\tilde{y}}+A(h \tilde{y})\right) u .
$$
In (1.8), (1.9) we can replace $u(y)$ by $v(x)=u\left(\frac{x}{h}\right)$, so the Hilbert space (1.8) can be
written as:

$$
\left\{v(x) \delta(x-h y), v \in L^{2}\left(\mathbb{R}_{x}^{n}\right)\right\}, \quad \text { with the norm } h^{-n / 2}\|v\|_{L^{2}\left(\mathbb{R}^{n}\right)} .
$$

Similarly the domain (1.9) can be written as:

$$
\begin{aligned}
& \left\{v(x) \delta(x-h y) ;\left(h D_{x}+A(x)\right)^{\alpha} v \in L^{2}\left(\mathbb{R}_{x}^{n}\right),|\alpha| \leqq m\right\} \\
& \text { with the norm } h^{-n / 2}\left(\sum_{|\alpha| \leqq m}\left\|\left(h D_{x}+A(x)\right)^{\alpha} u\right\|_{L^{2}\left(\mathbb{R}^{n}\right)}^{2}\right)^{1 / 2} .
\end{aligned}
$$

To further reduce the study of $P_{0}$, we apply the same method as for $P$.

The image of $v(x) \delta(x-h y)$ under the map $e^{i(x / h-y) \cdot \theta} \mathscr{U}$ is the distribution $v(x) \sum_{\gamma \in \Gamma} \delta(x-h y+h \gamma)$ which does not depend on $\theta$. From this we get that $P$ acting on (1.10) with domain (1.11) is unitarily equivalent to $P$ acting on

$$
L_{0} \stackrel{\text { def }}{=}\left\{\sum_{\gamma \in \Gamma} v(x) \delta(x-h y+h \gamma), v \in L^{2}\left(\mathbb{R}_{x}^{n}\right)\right\}
$$

with obtain

$$
L_{m} \underset{\operatorname{def}}{=}\left\{\sum_{\gamma \in \Gamma} v(x) \delta(x-h y+h \gamma)\left|\left(h D_{x}+A(x)\right)^{\alpha} v \in L^{2}\left(\mathbb{R}_{x}^{n}\right),\right| \alpha \mid \leqq m\right\} .
$$

Summing up, we have proved:

Proposition 1.3. $P_{0}=P^{w}\left(h y, y, D_{y}+A(h y)\right)$ acting on $L^{2}\left(\mathbb{R}_{y}^{n}\right)$ with domain $H_{m, A}$ is unitarily equivalent to $P=P^{w}\left(x, y, h D_{x}+D_{y}+A(x)\right)$ acting on $L_{0}$ with domain $L_{m}$. $P$ acting on $L_{0}$ will be further studied in Sect. III.

\section{Spectral Reduction of $P$}

In this section we consider more in detail the operator $P=P^{w}\left(x, y, D_{y}+h D_{x}+A(x)\right)$. We will give a reduction of the study of $\sigma(P)$ by considering $P$ as an $h$-pseudodifferential operator in the $x$ variables with an operator-valued symbol $\mathbb{P}(x, \xi+A(x))=P^{w}\left(x, y, D_{y}+\xi+A(x)\right)$, and by introducing a suitable "Grushin problem." A review of some basic results we will use about operator valued pseudodifferential operators is given in the Appendix, Sect. B.

To describe the estimates satisfied by some operator valued symbols, we 
introduce the following Hilbert spaces with their natural norms:

$$
\begin{aligned}
K_{0} & =L^{2}\left(\mathbb{R}_{y}^{n} / \Gamma\right), \\
K_{m, \xi} & =\left\{u \in K_{0}\left|\left(D_{y}+\xi\right)^{\alpha} u \in K_{0}, \forall\right| \alpha \mid \leqq m\right\} .
\end{aligned}
$$

We notice that only the norm on $K_{m, \xi}$ depends on $\xi$ and not the space itself and we have:

$$
\|u\|_{K_{m, \xi}} \leqq C\langle\xi-\eta\rangle^{m}\|u\|_{K_{m, \eta}}, \quad \forall u \in K_{m, 0}, \quad \xi, \eta \in \mathbb{R}^{n} .
$$

If we denote by $K_{m, \xi+A(x)}$ the same spaces obtained by replacing $\xi$ by $\xi+A(x)$, we see that (2.1) still holds if we replace $\langle\xi-\eta\rangle$ by $\langle\xi-\eta\rangle+\langle x-y\rangle$. (We use here hypothesis (H.5) on $A(x)$.) This means that the spaces $K_{m, \xi+A(x)}$ satisfy the assumptions of Appendix B.

Finally we notice that $\mathbb{P}(x, \xi+A(x))$ satisfies:

$$
\left\|\partial_{x}^{\alpha} \partial_{\xi}^{\beta} \mathbb{P}(x, \xi+A(x))\right\| \leqq C_{\alpha, \beta}
$$

where the norm \|\| is taken in $\mathscr{L}\left(K_{m, \xi+A(x)}, K_{0}\right)$.

To construct a suitable Grushin problem for $\mathbb{P}\left(x, h D_{x}+A(x)\right)$, the first step is to construct a Grushin problem on the symbolic level, i.e. for $\mathbb{P}(x, \xi+A(x))$. This is done in the next Proposition:

We will fix some energy level $\lambda_{0} \in \mathbb{R}^{+}$and denote by $\mathscr{F}_{0, \theta}$ the space $\left\{u \in L_{\text {loc }}^{2}\left(\mathbb{R}_{y}^{n}\right) \mid u(y+\gamma)=e^{i \gamma \cdot \theta} u(y)\right\}$ for $\theta \in \mathbb{R}^{n^{*}} / \Gamma^{*}$, and by $\mathscr{F}_{m, \theta}$ the space $\left\{u \in \mathscr{F}_{0, \theta} \mid D_{y}^{\alpha} u \in\right.$ $\left.\mathscr{F}_{0, \theta},|\alpha| \leqq m\right\}$.

Then we have the following Proposition:

Proposition 2.1. There exists $N \in \mathbb{N}$, a complex neighborhood $\mathscr{V}$ of $\lambda_{0}$, and functions $\varphi_{j}(x, y, \xi) \in C^{\infty}\left(\mathbb{R}_{x, \xi}^{2 n}, \mathscr{F}_{m, 0}\right) \cap C^{\infty}\left(\mathbb{R}_{x}^{n} \times \mathbb{R}_{\xi}^{n} \times \mathbb{R}_{y}^{n}\right)$ for $1 \leqq j \leqq N$, such that for each $(x, \xi) \in \mathbb{R}^{2 n}$, and each $\lambda \in \mathscr{V}$ the following operator:

$$
\mathscr{P}(x, \xi, \lambda)\left(\begin{array}{c}
u \\
u^{-}
\end{array}\right)=\left(\begin{array}{c}
\left(P^{w}\left(x, y, D_{y}+\xi\right)-\lambda\right) u+R_{-}(x, \xi) u^{-} \\
R_{+}(x, \xi) u
\end{array}\right)
$$

is invertible from $K_{m, \xi} \oplus \mathbb{C}^{N}$ into $K_{0} \oplus \mathbb{C}^{N}$ with an inverse $\mathscr{E}_{0}(x, \xi, \lambda)$ uniformly bounded with respect to $(x, \xi, \lambda)$ together with all derivatives in $\mathscr{L}\left(K_{0} \times \mathbb{C}^{N}, K_{m, \xi} \times \mathbb{C}^{N}\right)$ for $(x, \xi) \in \mathbb{R}^{2 n}, \lambda \in \mathscr{V}$. Here $\left(R_{+}(x, \xi) u\right)_{j}=\left\langle u, \varphi_{j}(x, \cdot \xi)\right\rangle_{\mathscr{F}_{0,0}}$ and $R_{-}(x, \xi) u^{-}=$ $\sum_{j=1}^{N} u_{j}^{-} \varphi_{j}(x, \cdot, \xi)$.

Moreover the functions $\varphi_{j}$ satisfy the estimates:

$$
\left\{\begin{array}{l}
\left\|\partial_{x}^{\alpha} \partial_{\xi}^{\beta} \varphi_{j}\right\|_{\boldsymbol{K}_{m, \xi}} \leqq C_{\alpha, \beta} \forall \alpha, \beta \in \mathbb{N}^{n}, x, \xi \in \mathbb{R}^{n} \\
\forall \gamma^{*} \in \Gamma^{*}, \varphi_{j}\left(x, y, \xi+\gamma^{*}\right)=e^{-i y \gamma^{*}} \varphi_{j}(x, y, \xi) .
\end{array}\right.
$$

Remark. In fact, we shall see in the proof that we can even choose the $\varphi_{j}$ 's independent of $x$. But sometimes it can be convenient to use $\varphi_{j}$ 's depending also on $x$. In the proposition, it is also possible to let $\lambda_{0}$ be a compact interval instead of a number.

Proof. We will follow the ideas of $[\mathrm{He}-\mathrm{Sj} 1] \mathrm{Sect}$. 3.

In fact for $\lambda=\lambda_{1}$ fixed, Theorem 3.1 of [He-Sj1] gives the existence of $N$ 
analytic sections $\psi_{j}(y, \theta)$ of the bundle with base $\mathbb{R}^{n^{*}} / \Gamma^{*}$ and with fiber over $\theta$ equal to $\mathscr{F}_{0, \theta}$, having the following property:

The Grushin problem

$$
\left\{\begin{array}{l}
\left((-\Delta)^{m / 2}-\lambda_{1}\right) u+R_{\theta}^{-} u=v \\
R_{\theta}^{+} u=v^{+}
\end{array}\right.
$$

is bijective from $\mathscr{F}_{m, \theta} \times \mathbb{C}^{N}$ into $\mathscr{F}_{0, \theta} \times \mathbb{C}^{N}$ if we take:

$$
\left(R_{\theta}^{+} u\right)_{j}=\left\langle u, \psi_{j}(\cdot, \theta)\right\rangle_{\mathscr{F}_{0, \theta}} \text { and } R_{\theta}^{-} u^{-}=\sum_{j=1}^{N} u_{j}^{-} \psi_{j}(\cdot, \theta) .
$$

More precisely, we have an a priori inequality of the following type:

$$
\exists C_{0}>0 \text { such that } \forall u \in \mathscr{F}_{m, \theta} \cap \operatorname{Vect}\left(\psi_{1}, \ldots, \psi_{N}\right)^{\perp}
$$

(here ()$^{\perp}$ is the orthogonal subspace with respect to $\mathscr{F}_{0, \theta}$ ), one has:

$$
\left\langle\left(\left((-\Delta)^{m / 2}-\lambda_{1}\right) u, u\right\rangle_{\mathscr{F}_{0, \theta}} \geqq \frac{1}{C_{0}}\|u\|_{\mathscr{F}_{0, \theta}}^{2} .\right.
$$

Using hypotheses (H.3), (H.4), we get by standard inequalities:

$$
\left\langle P^{w}\left(x, y, D_{y}\right) u, u\right\rangle_{\mathscr{F}_{0}, \theta} \geqq \frac{1}{C_{1}}\left\langle(-\Delta)^{m / 2} u, u\right\rangle_{\mathscr{F}_{0, \theta}}-C_{1}\|u\|_{\mathscr{F}_{0, \theta}}^{2},
$$

uniformly for $x \in \mathbb{R}^{n}, u \in \mathscr{F}_{m, \theta}$.

Hence for $u \in \operatorname{Vect}\left(\psi_{1}, \ldots, \psi_{N}\right)^{\perp}$ and $\lambda \in \mathscr{V}$ we get:

$$
\operatorname{Re}\left\langle\left(P^{w}\left(x, y, D_{y}\right)-\lambda\right) u, u\right\rangle_{\mathscr{F}_{0}, \theta} \geqq\left(\frac{1}{C_{0} C_{1}}+\frac{\lambda_{1}}{C_{1}}-\operatorname{Re} \lambda-C_{1}\right)\|u\|_{\mathscr{F}_{0}, \theta}^{2} \cdot
$$

If we take $\lambda_{1}$ big enough (and let $N$ increase correspondingly) we get:

$$
\operatorname{Re}\left\langle\left(P^{w}\left(x, y, D_{y}\right)-\lambda\right) u, u\right\rangle_{\mathscr{F}_{0, \theta}} \geqq \frac{1}{C_{2}}\|u\|_{\mathscr{F}_{0, \theta}}^{2}
$$

with $C_{2}>0$, uniformly for $x \in \mathbb{R}^{n}, \lambda \in \mathscr{V}, \theta \in \mathbb{R}^{n^{*}} / \Gamma^{*}$ and $u \in \mathscr{F}_{m, \theta} \cap \operatorname{Vect}\left(\psi_{1}, \ldots, \psi_{N}\right)^{\perp}$.

It is easy to see that $(2.5)$ implies that the Grushin problem $(2.2)^{\prime}$ with $(-\Delta)^{m / 2}$ replaced by $P^{w}\left(x, y, D_{y}\right)$ is bijective from $\mathscr{F}_{m, \boldsymbol{\theta}} \times \mathbb{C}^{N}$ into $\mathscr{F}_{0, \boldsymbol{\theta}} \times \mathbb{C}^{N}$ with an inverse uniformly bounded for $x \in \mathbb{R}^{n}, \theta \in \mathbb{R}^{n^{*}} / \Gamma^{*}, \lambda \in \mathscr{V}$.

Moreover the $\psi_{j}$ constructed in $[\mathrm{He}-\mathrm{Sj} 1]$ are of the form:

$$
\psi_{j}(y, \theta)=\sum_{\gamma \in \Gamma} \phi_{j}(y-\gamma) e^{i \theta \cdot \gamma}
$$

with $\phi_{j}(y) \in C_{0}^{\infty}(E)$ where $E$ is a fundamental domain of $\mathbb{R}^{n} / \Gamma$, and the $\phi_{j}$ 's are linearly independent.

Then if we take $\varphi_{j}(y, \xi)=\psi_{j}(y, \xi) e^{-i y \cdot \xi}$, it is easy to see that the Grushin problem in Proposition 2.1 is invertible, with an inverse $\mathscr{E}_{0}$ uniformly bounded in $\mathscr{L}\left(K_{0} \times \mathbb{C}^{N}, K_{m, \xi} \times \mathbb{C}^{N}\right)$. Indeed this follows from the fact that $u \mapsto e^{-i y \cdot \xi} u$ is unitary from $\mathscr{F}_{m, \xi}$ into $K_{m, \xi}$.

The estimates on $\partial_{x}^{\alpha} \partial_{\xi}^{\beta} \mathscr{E}_{0}$ follow easily from similar estimates on $\partial_{x}^{\alpha} \partial_{\xi}^{\beta} \mathscr{P}$, and the properties $(2.1)^{\prime}$ on $\varphi_{j}$ are immediate consequences of the above remark. 
From now on, we denote by $\mathscr{P}(x, \xi, \lambda)$ any operator constructed as in Proposition 2.1 with functions $\varphi_{j}$ 's satisfying (2.1)'. We also denote by $\left(\begin{array}{cc}E_{0}(x, \xi, \lambda) & E_{0}^{+}(x, \xi, \lambda) \\ E_{0}^{-}(x, \xi, \lambda) & E_{0}^{-+}(x, \xi, \lambda)\end{array}\right)$ the matrix elements of $\mathscr{E}_{0}(x, \xi, \lambda)$. We now turn to the quantization of $\mathscr{P}(x, \xi, \lambda)$ and $\mathscr{E}_{0}(x, \xi, \lambda)$.

Proposition 2.2. The operator $\mathscr{E}_{0}^{w}\left(x, h D_{x}+A(x), \lambda\right)$ is continuous from $\mathscr{S}\left(\mathbb{R}^{n}, K_{0} \oplus \mathbb{C}^{N}\right)$ into $\mathscr{S}\left(\mathbb{R}^{n}, K_{m, \xi+A(x)} \oplus \mathbb{C}^{N}\right)$, from $S^{\prime}\left(\mathbb{R}^{n}, K_{0} \oplus \mathbb{C}^{N}\right)$ into $\mathscr{S}^{\prime}\left(\mathbb{R}^{n}, K_{m, \xi+A(x)} \oplus \mathbb{C}^{N}\right)$ and uniformly bounded from $L^{2}\left(\mathbb{R}_{x}^{n} \times \mathbb{R}_{y}^{n} / \Gamma\right) \oplus L^{2}\left(\mathbb{R}_{x}^{n}, \mathbb{C}^{N}\right)$ into $\mathscr{K}_{m} \oplus L^{2}\left(\mathbb{R}_{x}^{n}, \mathbb{C}^{N}\right)$ for $\lambda \in \mathscr{V}$ and $0<h \leqq 1$.

Moreover we have:

$$
\mathscr{P}^{w}\left(x, h D_{x}+A(x), \lambda\right) \circ \mathscr{E}_{0}^{w}\left(x, h D_{x}+A(x), \lambda\right)=1+h \mathscr{R}^{w}\left(x, h D_{x}+A(x), \lambda, h\right),
$$

where $\partial_{\lambda}^{k} \mathscr{R}(x, \xi, \lambda, h) \in S^{0}\left(\mathbb{R}^{i 2 n}, \mathscr{L}\left(K_{0} \oplus \mathbb{C}^{N}, K_{0} \times \mathbb{C}^{N}\right)\right) \forall k \in \mathbb{N}$, and: $\mathscr{R}(x, \xi, \lambda, h)$ has an asymptotic expansion $\mathscr{R}_{0}(x, \xi, \lambda)+h \mathscr{R}_{1}(x, \xi, \lambda)+\cdots$ uniformly with respect to $\lambda \in \mathscr{V}$, with $\partial_{\lambda}^{k} \mathscr{R}_{j} \in S^{0}\left(\mathbb{R}^{2 n}, \mathscr{L}\left(K_{0} \oplus \mathbb{C}^{N}, K_{0} \oplus \mathbb{C}^{N}\right)\right)$.

Proof. The continuity of $\mathscr{E}_{0}^{w}\left(x, h D_{x}+A(x), \lambda\right)$ in $\mathscr{S}$ and $\mathscr{S}^{\prime}$ follows from the calculus of operator valued p.d.o's established in Appendix B, where (2.7) is also obtained.

It remains to prove the $L^{2}$ boundedness statement. For that, we remark using the results of Appendix A (more precisely formulas (A.1), (A.2)) that $\mathscr{K}_{m}$ can be described as

$$
\mathscr{K}_{m}=\left\{y \in K_{0}\left|\left(\left(h D_{x}+A(x)+D_{y}\right)^{\alpha}\right)^{w} u \in \mathscr{K}_{0},\right| \alpha \mid \leqq m\right\},
$$

(where by $\left(\left(h D_{x}+A(x)+D_{y}\right)^{\alpha}\right)^{w}$ we mean the quantization of $\left.(h \xi+A(x)+\eta)^{\alpha}\right)$, with equivalent norms. One can also view $\left(\left(h D_{x}+A(x)+D_{y}\right)^{\alpha}\right)^{w}$ as an $h$-pseudodifferential operator with the operator valued symbol $\left(\left(\xi+A(x)+D_{y}\right)^{\alpha}\right)^{w}: K_{m, \xi+A(x)} \rightarrow K_{0}$. From the composition and $L^{2}$-boundedness results of Appendix $\mathrm{B}$, we get that, since

$$
\left(\begin{array}{cc}
\left(\left(\xi+A(x)+D_{y}\right)^{\alpha}\right)^{w} & 0 \\
0 & 1
\end{array}\right)\left(\begin{array}{cc}
E_{0}(x, \xi+A(x), \lambda) & E_{0}^{+}(x, \xi+A(x), \lambda) \\
E_{0}^{-}(x, \xi+A(x), \lambda) & E_{0}^{-+}(x, \xi+A(x), \lambda)
\end{array}\right)
$$

is in $S^{0}\left(\mathbb{R}^{2 n}, \mathscr{L}\left(K_{0} \times \mathbb{C}^{N}, K_{0} \times \mathbb{C}^{N}\right)\right)$,

$$
\left(\begin{array}{cc}
\left(\left(h D_{x}+A(x)+D_{y}\right)^{\alpha}\right)^{w} & 0 \\
0 & 1
\end{array}\right) \circ \mathscr{E}_{0}^{w}\left(x, h D_{x}+A(x), \lambda\right)
$$

is $0(1)$ in $\mathscr{L}\left(\mathscr{K}_{0} \oplus L^{2}\left(\mathbb{R}_{x}^{n}, \mathbb{C}^{N}\right), \mathscr{K}_{0} \oplus L^{2}\left(\mathbb{R}_{x}^{n}, \mathbb{C}^{N}\right)\right)$, which proves the Proposition.

We can now use R. Beals' characterisation of pseudodifferential operators (cf. [Be]) with symbols in $S^{0}$, in the semiclassical version of [He-Sj2] and with operator valued symbols, to see that for $h>0$ small enough:

$$
\left(\mathbf{1}+h \mathscr{R}^{w}\left(x, h D_{x}+A(x), \lambda, h\right)\right)^{-1}=\mathbf{1}+h \tilde{\mathscr{R}}^{w}\left(x, h D_{x}+A(x), \lambda, h\right),
$$

where $\tilde{\mathscr{R}}^{w}$ is an $h$-pseudodifferential operator with the same properties as $\mathscr{R}^{w}$.

We then replace $\mathscr{E}_{0}^{w}\left(x, h D_{x}+A(x), \lambda\right)$ by $\mathscr{E}_{0}^{w}\left(x, h D_{x}+A(x), \lambda\right) \circ\left(1+h \tilde{\mathscr{R}}^{w}\right)$, which we can write $\mathscr{E}^{w}\left(x, h D_{x}+A(x), \lambda, h\right)$ or $\mathscr{E}^{w}$ for simplicity.

Summing up, we have proved a part of the following result:

Theorem 2.3. Assume (H1) to (H5).

For $h$ sufficiently small and $\lambda \in \mathscr{V}, \mathscr{P}^{w}\left(x, h D_{x}+A(x), \lambda\right)$ has a uniformly bounded 
inverse of the form $\mathscr{E}^{w}\left(x, h D_{x}+A(x), \lambda, h\right)$, where

$$
\mathscr{E}(\dot{x}, \xi, \lambda, h) \in S^{0}\left(\mathbb{R}^{2 n}, \mathscr{L}\left(K_{0} \oplus \mathbb{C}^{N}, K_{m, \xi} \oplus \mathbb{C}^{N}\right)\right)
$$
has an asymptotic expansion $\mathscr{E}^{w}(x, \xi, \lambda, h) \sim \sum_{0}^{\infty} \mathscr{E}_{j}(x, \xi, \lambda) h^{j}, \mathscr{E}_{0}=\mathscr{P}(x, \xi, \lambda)^{-1}$ as
above.

This inverse has the same continuity properties as $\mathscr{E}_{0}\left(x, h D_{x}+A(x), \lambda, h\right)$ in Proposition 2.2.

Proof. From the constructions above it is clear that $\mathscr{E}=\mathscr{E}^{w}\left(x, h D_{x}+A(x), \lambda, h\right)$ is a right inverse for $\mathscr{P}^{w}\left(x, h D_{x}+A(x), \lambda\right)$ with all the properties stated in the Theorem. We only have to show that $\mathscr{E}$ is also a left inverse. If $\lambda \in \mathscr{V} \cap \mathbb{R}, \mathscr{P}^{w}\left(x, h D_{x}+A(x), \lambda\right)$ is self adjoint on $K_{0} \oplus L^{2}\left(\mathbb{R}^{n}, \mathbb{C}^{N}\right)$ with domain $K_{m} \oplus L^{2}\left(\mathbb{R}^{n}, \mathbb{C}^{N}\right)$. Then $\mathscr{E}^{w}\left(x, h D_{x}+A(x), \lambda, h\right)$ is also a left inverse for $\lambda \in \mathscr{V} \cap \mathbb{R}$, and also for $\lambda \in \mathscr{V}$ by analytic continuation.

This proves the theorem.

We denote by $\left(\begin{array}{cc}E(\lambda, h) & E_{+}(\lambda, h) \\ E_{-}(\lambda, h) & E_{-+}(\lambda, h)\end{array}\right)$ the matrix elements of $\mathscr{E}$ and remark the $E_{-+}(\lambda, h)=E_{-+}^{w}\left(x, h D_{x}+A(x), \lambda, h\right)$ is a $h$-pseudodifferential operator with its symbol in $S^{0}\left(\mathbb{R}^{2 n}, \mathscr{L}\left(\mathbb{C}^{N}, \mathbb{C}^{N}\right)\right)$. In particular $E_{-+}(\lambda, h)$ is bounded on $L^{2}\left(\mathbb{R}^{n}, \mathbb{C}^{N}\right)$.

We come now to the main result of this section, namely the reduction of the spectral study of $P$ :

Theorem 2.4. Under assumptions (H1) to (H.5), for $\lambda \in \mathscr{V}, h$ small enough, one has the following equivalence:

$$
\lambda \in \sigma(P) \Leftrightarrow 0 \in \sigma\left(E_{-+}(\lambda, h)\right) .
$$

Proof. We use the following formulas which can be checked easily:

$$
\begin{gathered}
(P-\lambda)^{-1}=E(\lambda, h)-E_{+}(\lambda, h) E_{-+}(\lambda, h)^{-1} E_{-}(\lambda, h), \\
E_{-+}(\lambda, h)^{-1}=-R_{+}^{w}\left(x, h D_{x}+A(x)\right)(P-\lambda)^{-1} R_{-}^{w}\left(x, h D_{x}+A(x)\right) .
\end{gathered}
$$

Here $R_{ \pm}^{w}\left(x, h D_{x}+A(x)\right)$ is the Weyl quantization of $R_{ \pm}(x, h \xi+A(x))$ in Proposition 2.1. Then (2.8), (2.9) and the continuity properties of $\mathscr{E}$ established in Theorem 2.3 imply Theorem 2.4 .

As a preparation for the next section, we will now establish some commutation properties of $\mathscr{E}$.

Because of $(2.1)^{\prime}$, we have:

$$
\left\{\begin{array}{l}
R_{-}\left(x, \xi+\gamma^{*}\right)=e^{-i y \cdot \gamma^{*}} R_{-}(x, \xi) \\
R_{+}\left(x, \xi+\gamma^{*}\right)=R_{+}(x, \xi) e^{i y \cdot \gamma^{*}}
\end{array}\right.
$$

On the operator level we get:

$$
\begin{aligned}
& e^{-i x \cdot \gamma^{*} / h} R_{-}^{w}\left(x, h D_{x}+A(x)\right) e^{i x \cdot \gamma^{*} / h}=e^{-i y \cdot \gamma^{*}} R_{-}^{w}\left(x, h D_{x}+A(x)\right), \\
& e^{-i x \cdot \gamma^{*} / h} R_{+}^{w}\left(x, h D_{x}+A(x)\right) e^{i x \cdot \gamma^{*} / h}=R_{+}^{w}\left(x, h D_{x}+A(x)\right) e^{i y \cdot \gamma^{*}}
\end{aligned}
$$

Combining this with the fact that

$$
P^{w}\left(x, y, \xi+\gamma^{*}+D_{y}\right)=e^{-i y \cdot \gamma^{*}} P^{w}\left(x, y, \xi+D_{y}\right) e^{i y \cdot \gamma^{*}},
$$


we get

$$
e^{-i x \cdot \gamma^{*} / h} \mathscr{P}^{w}\left(x, h D_{x}+A(x)\right) e^{i x \cdot \gamma^{*} / h}=\left(\begin{array}{cc}
e^{-i y \cdot \gamma^{*}} & 0 \\
0 & 1
\end{array}\right) \mathscr{P}^{w}\left(x, h D_{x}+A(x)\right)\left(\begin{array}{cc}
e^{i y \cdot \gamma^{*}} & 0 \\
0 & 1
\end{array}\right)
$$

This can be rewritten as:

$$
\left[\mathscr{P}^{w}\left(x, h D_{x}+A(x)\right),\left(\begin{array}{cc}
e^{i(x / h-y) \cdot \gamma^{*}} & 0 \\
0 & e^{i x \cdot \gamma^{*} / h}
\end{array}\right)\right]=0 .
$$

Of course (2.11) stays true if we replace $\mathscr{P}^{w}\left(x, h D_{x}+A(x)\right)$ by its inverse $\mathscr{E}$, which is what we will need in the next section.

\section{Spectral Reduction of $\boldsymbol{P}_{\mathbf{0}}$}

In this section, we give a reduction of the study of the spectrum of $P_{0}$ by using Proposition 1.3. Hence we replace $P_{0}$ by $P$ acting on $L_{0}$ with domain $L_{m}$ (see Sect. I) and we shall use the Grushin problem of Sect. II to study this operator.

First, let us remark that a distribution $u=\sum_{\gamma \in \Gamma} v(x) \delta(x-h(y-\gamma))$ in $L_{0}$ can also be written as $\sum_{\gamma \in \Gamma} \delta(x-h(y-\gamma)) v(h(y-\gamma))$, i.e. as an element of $\mathscr{S}^{\prime}\left(\mathbb{R}_{x}^{n} ; K_{0}\right)$ where, as before, $K_{0}=L^{2}\left(\mathbb{R}^{n} / \Gamma\right)$ : indeed, if

$$
\varphi \in \mathscr{S}\left(\mathbb{R}^{n}, K_{0}\right) \cong\left\{\langle x\rangle^{N} \partial_{x}^{\alpha} \varphi \in L^{2}\left(\mathbb{R}^{n} \times F\right), \forall N, \alpha\right\}
$$

(where $F$ is a fundamental domain of $\left.\mathbb{R}^{n} / \Gamma\right)$, then $\tilde{\varphi}(\tilde{x}, \tilde{y}) \stackrel{\text { def }}{=} \varphi(\tilde{x}+h \tilde{y}, \tilde{y})$ is also in $\mathscr{S}\left(\mathbb{R}^{n}, K_{0}\right)$, and we have:

$$
\langle u, \varphi\rangle=\sum_{\gamma \in \Gamma} \int_{\boldsymbol{F}} v(h(\tilde{y}-\gamma)) \tilde{\varphi}(-h \gamma, \tilde{y}) d \tilde{y}
$$

which can be bounded by seminorms of $\tilde{\varphi}$ in $\mathscr{S}\left(\mathbb{R}^{n}, K_{0}\right)$, and thus also by seminorms of $\varphi$ in $\mathscr{S}\left(\mathbb{R}^{n}, K_{0}\right)$.

Hence, we can hope to adapt some results of Sect. II for the study of $P$ acting on $L_{0}$.

Let us denote by $V_{0}$ the subspace of $\mathscr{S}^{\prime}\left(\mathbb{R}_{x}^{n}\right)$ consisting of the distributions of the form:

$$
w(x)=\sum_{\gamma \in \Gamma} f_{\gamma} \delta(x-h \gamma)
$$

with $\left(f_{\gamma}\right)_{\gamma \in \Gamma} \in \ell^{2}(\Gamma) . V_{0}$ is equipped with its natural Hilbert space structure, and we first study this space in more detail. Let $\tau_{\gamma^{*}}$ be the operator of multiplication by $e^{i x \cdot \gamma^{*} / h}$. We have:

Proposition 3.1. Let $\chi \in S^{0}\left(\mathbb{R}^{2 n}\right)$ be such that there exists a compact set $K$ of $\mathbb{R}^{n}$ with $\operatorname{supp} \chi \subset \mathbb{R}_{x}^{n} \times K$, and $\sum_{\gamma^{*} \in \Gamma^{*}} \chi\left(x, \xi+\gamma^{*}\right)=1$.

Then, for $u, v \in V_{0}$, the quantity $\left(\chi^{w}\left(x, h D_{x}\right) u, v\right)_{L^{2}\left(\mathbb{R}^{n)}\right.}$ is finite, independent of the choice of $\chi$, and satisfies:

$$
\left(\chi^{w}\left(x, h D_{x}\right) u, v\right)_{L^{2}\left(\mathbb{R}^{n}\right)}=\frac{1}{h^{n} \operatorname{Vol}\left(\mathbb{R}^{n} / \Gamma\right)}\langle u, v\rangle_{V_{0}} .
$$


Proof. We have:

$$
\left(\chi^{w}\left(x, h D_{x}\right) u, v\right)_{L^{2}}=\sum_{\gamma, \gamma^{\prime}} f_{u}(\gamma) \overline{f_{v}\left(\gamma^{\prime}\right)}\left(\chi^{w}\left(x, h D_{x}\right) \delta(\cdot-h \gamma), \delta\left(\cdot-h \gamma^{\prime}\right)\right)_{L^{2}}
$$

where $f_{u}(\gamma), f_{v}\left(\gamma^{\prime}\right)$ denote the coefficients in (3.1) for $u$ and $v$.

Thus

$$
\left(\chi^{w}\left(x, h D_{x}\right) u, v\right)_{L^{2}}=(2 \pi h)^{-n} \sum_{\gamma, \gamma^{\prime}} f_{u}(\gamma) \overline{f_{v}\left(\gamma^{\prime}\right)} \rho\left(\gamma, \gamma^{\prime}\right)
$$

with

$$
\begin{aligned}
\rho\left(\gamma, \gamma^{\prime}\right) & =\int e^{i\left(\gamma^{\prime}-\gamma\right) \xi} \chi\left(h \frac{\gamma+\gamma^{\prime}}{2}, \xi\right) d \xi \\
& =\sum_{\gamma^{*} F^{*}} e^{i\left(\gamma^{\prime}-\gamma\right) \xi} \chi\left(h \frac{\gamma+\gamma^{\prime}}{2}, \xi+\gamma^{*}\right) d \xi,
\end{aligned}
$$

where $F^{*}$ denotes a fundamental domain of $\mathbb{R}^{n^{*}} / \Gamma^{*}$.

Since $\sum_{\gamma^{*}} \chi\left(x, \xi+\gamma^{*}\right)=1$ for any $x, \xi$, we then get:

$$
\begin{aligned}
\left(\chi^{w}\left(x, h D_{x}\right) u, v\right)_{L^{2}} & =\frac{\operatorname{Vol}\left(\mathbb{R}^{n^{*}} / \Gamma^{*}\right)}{(2 \pi h)^{n}} \sum_{\gamma} f_{u}(\gamma) \overline{f_{v}(\gamma)} \\
& =\frac{1}{h^{n} \operatorname{Vol}\left(\mathbb{R}^{n} / \Gamma\right)}\langle u, v\rangle_{V_{0}} .
\end{aligned}
$$

We now give another characterisation of $V_{0}$ :

Proposition 3.2. For every $u \in V_{0}$, there exists $u_{0} \in L^{2}\left(\mathbb{R}^{n}\right)$ such that:

If $u_{\gamma^{*}} \stackrel{\text { def }}{=} \tau_{\gamma^{*}} u_{0}$, then $u=\sum_{\gamma^{*} \in \Gamma^{*}} u_{\gamma^{*}}$ where the sum converges in $\mathscr{S}^{\prime}\left(\mathbb{R}^{n}\right)$.

$\exists C>0$ such that for any bounded set $\mathscr{B}$ in $S^{0}\left(\mathbb{R}^{2 n}\right)$ and

any $N \in \mathbb{N}$, there exists a constant $C_{N}>0$ such that

$\forall A \in \mathscr{B}$ with $\operatorname{dist}\left(\operatorname{Supp} A, \mathbb{R}_{x}^{n} \times\{0\}\right) \geqq C$ we have,

$\left\|A^{w}\left(x, h D_{x}\right) u_{0}\right\|_{L_{2}\left(\mathbb{R}^{n}\right)} \leqq C_{N} h^{N}\left(\operatorname{dist}\left(\operatorname{Supp} A, \mathbb{R}^{n} \times\{0\}\right)\right)^{-N}$

uniformly for $h>0$ small enough.

Moreover, the constants $C_{N}$ can be taken $\mathcal{O}\left(\|u\|_{V_{0}}\right)$ uniformly with respect to $u$.

Conversely, if $u_{0} \in L^{2}\left(\mathbb{R}^{n}\right)$ satisfies (3.3) and if $u_{\gamma^{*}}=\tau_{-\gamma^{*}} u_{0}$, then the sum $\sum_{\gamma^{*} \in \Gamma^{*}} u_{\gamma^{*}}$ converges in $\mathscr{S}^{\prime}(\mathbb{R})$ towards an element $u$ of $V_{0}$, with $\|u\|_{V_{0}}$ bounded by a constant times $h^{n / 2}$ times the sum of $\left\|u_{0}\right\|_{L^{2}}$ and a finite number of the $C_{N}$ 's in (3.3).

Proof. If $u \in V_{0}$, we take $u_{0}=\chi\left(h D_{x}\right) u$ with $\chi \in C_{0}^{\infty}\left(\mathbb{R}^{n}\right), \sum_{\gamma^{*}} \chi\left(\xi+\gamma^{*}\right)=1$. Then $u_{0}=(2 \pi h)^{-n} \sum_{\gamma \in \Gamma} f_{u}(\gamma) \hat{\chi}\left(\gamma-\frac{x}{h}\right)$ is in $H^{s}\left(\mathbb{R}^{n}\right)$ for any $s$ (here $\left.\hat{\chi}(x)=\int e^{-i x \xi} \chi(\xi) d \xi\right)$, and satisfies:

$$
\forall \alpha \in \mathbb{N}^{n}, \quad\left\|\left(h D_{x}\right)^{\alpha} u_{0}\right\|_{L^{2}\left(\mathbb{R}^{n}\right)} \leqq C_{\alpha} h^{-n / 2}\|u\|_{V_{0}}
$$


The property (3.3) follows then, by integrating by parts in the oscillatory integral which gives $A^{w}\left(x, h D_{x}\right) u_{0}$, and using the Calderon-Vaillancourt theorem.

To prove (3.2), we only remark that $u_{\gamma^{*}}=\tau_{\gamma^{*}} \chi\left(h D_{x}\right) \tau_{-\gamma^{*}} u=\chi\left(h D_{x}-\gamma^{*}\right) u$.

Let us now prove the converse statement:

If $u_{0}$ satisfies (3.3), we can find for any $\gamma^{*} \in \Gamma^{*}$ a real function $A_{\gamma^{*}}(\xi) \in C_{0}^{\infty}\left(|\xi|<\frac{1}{2}\left|\gamma^{*}\right|\right)$ such that the family $\left(A_{\gamma^{*}}\right)_{\gamma^{*} \in \Gamma^{*}}$ is bounded in $S^{0}\left(\mathbb{R}^{2 n}\right)$ and

$$
\left\|\left(1-A_{\gamma^{*}}\left(h D_{x}\right)\right) u_{0}\right\|_{L^{2}} \leqq C_{N}\left|\gamma^{*}\right|^{-N} h^{N}
$$

for $\left|\gamma^{*}\right|$ large enough. Then, if $\varphi \in \mathscr{S}\left(\mathbb{R}^{n}\right)$, we can write

$$
\left(u_{\gamma^{*}}, \varphi\right)_{L^{2}}=\left(u_{0}, \tau_{-\gamma^{*}} \varphi\right)_{L^{2}}=\left(u_{0}, A_{\gamma^{*}}\left(h D_{x}\right) \tau_{-\gamma^{*}} \varphi\right)_{L^{2}}+\mathcal{O}\left(h^{N}\left|\gamma^{*}\right|^{-N}\right)
$$

and, because of the condition on the support of $A_{\gamma^{*}}$, we have

$$
\left\|A_{\gamma^{*}}\left(h D_{x}\right) \tau_{-\gamma^{*}} \varphi\right\|_{L^{2}}=\mathcal{O}\left(h^{N}\left|\gamma^{*}\right|^{-N}\right) \text {. }
$$

We conclude from this that $\sum u_{\gamma^{*}}$ converges in $\mathscr{S}^{\prime}$. It remains to show that $u=\sum u_{\gamma^{*}}$ is in $V_{0}$.

Let us take $\chi$ as before, and consider

$$
\left(\chi\left(h D_{x}\right) u_{\alpha^{*}}, u_{\beta^{*}}\right)_{L^{2}}=\left(\chi\left(h D_{x}+\alpha^{*}\right) u_{0}, u_{\beta^{*}-\alpha^{*}}\right)_{L^{2}} .
$$

If we take another cutoff function $\tilde{\chi}$ such that $\tilde{\chi} \chi=\chi$, we get:

$$
\begin{aligned}
\left(\chi\left(h D_{x}\right) u_{\alpha^{*}}, u_{\beta^{*}}\right)_{L^{2}} & =\left(\chi\left(h D_{x}+\alpha^{*}\right) u_{0}, \tilde{\chi}\left(h D_{x}+\alpha^{*}\right) u_{\beta^{*}-\alpha^{*}}\right) \\
& =\left(\tau_{\alpha^{*}-\beta^{*}} \chi\left(h D_{x}+\alpha^{*}\right) u_{0}, \tilde{\chi}\left(h D_{x}+\beta^{*}\right) u_{0}\right) .
\end{aligned}
$$

Hence, using (3.3), we get:

$$
\begin{aligned}
\left|\left(\chi\left(h D_{x}\right) u_{\alpha^{*}}, u_{\beta^{*}}\right)_{L^{2}}\right| & \leqq\left\|\chi\left(h D_{x}+\alpha^{*}\right) u_{0}\right\|\left\|\tilde{\chi}\left(h D_{x}+\beta^{*}\right) u_{0}\right\| \\
& \leqq \tilde{C}_{N} h^{N}\left(\left|\alpha^{*}\right|+\left|\beta^{*}\right|\right)^{-N}
\end{aligned}
$$

when $\left|\alpha^{*}\right|+\left|\beta^{*}\right|$ is sufficiently large. Here, $\tilde{C}_{N}$ can be estimated by the sum of $\left\|u_{0}\right\|$ and a finite number of the $C_{N}$ 's in (3.3).

It follows from $(3.5)$ that $\left(\chi\left(h D_{x}\right) u, u\right)_{L^{2}}$ is well defined and finite, and we conclude by Proposition 3.1 that $u$ in $V_{0}$.

We next study the action of pseudodifferential operators on $V_{0}$ :

Proposition 3.3. Let $B(x, \xi) \in S^{0}\left(\mathbb{R}^{2 n}\right)$ with $B\left(x, \xi+\gamma^{*}\right)=B(x, \xi)$ for any $\gamma^{*} \in \Gamma^{*}$. Then, $B^{w}\left(x, h D_{x}\right)$ is bounded on $V_{0}$, uniformly with respect to $h$ small enough.

Proof. Let $u \in V_{0}$ and let us write $u=\sum_{\Gamma^{*}} u_{\gamma^{*}}$ as in Proposition 3.2. Then $B^{w}\left(x, h D_{x}\right) u=\sum_{\Gamma^{*}} v_{\gamma^{*}}$, where $v_{\gamma^{*}}=B^{w}\left(x, h D_{x}\right) u_{\gamma^{*}}$.

Then since $B\left(x, \xi+\gamma^{*}\right)=B(x, \xi)$, we get that $v_{\gamma^{*}}=\tau_{-\gamma^{*}} v_{0}$, where $v_{0}=B^{w}\left(x, h D_{x}\right) u_{0}$ is in $L^{2}\left(\mathbb{R}^{n}\right)$. This shows that $v_{\gamma^{*}}$ satisfy (3.2).

Using standard pseudodifferential operator calculus, we see easily that $v_{0}$ satisfies (3.3), with constants $C_{N}$ estimated by similar constants for $u_{0}$. This proves that $v \in V_{0}$ and that $\|v\|_{V_{0}} \leqq C_{0}\|u\|_{V_{0}}$, by Proposition 3.2.

Remark 3.4. An alternative proof of it would have been to conjugate $B^{w}\left(x, h D_{x}\right)$ by a Fourier transform, and then get a pseudodifferential operator acting on $L^{2}\left(\mathbb{R}^{n^{*}} / \Gamma^{*}\right)$. However, this kind of proof cannot be easily generalized to the space $L_{0}$ we have now to consider. 
Remark 3.5. Under the assumptions of Proposition 3.3, the adjoint of $B^{w}\left(x, h D_{x}\right)$ on $V_{0}$ is given by $(\bar{B})^{w}\left(x, h D_{x}\right)$.

We will now see that essentially the same discussion applies to $L_{0}$. Indeed if $u=\sum_{\gamma \in \Gamma} v(x) \delta(x-h y-h \gamma)$, we can consider for each fixed $y$ :

$$
u_{y}=\sum_{\gamma \in \Gamma} v(h y+h \gamma) \delta(x-h y-h \gamma)
$$

It turns out that up to the translation by $h y, u_{y}$ is an element of $V_{0}$ for almost all $y$. This follows from the following identity:

$$
\begin{aligned}
\|u\|_{L_{0}}^{2}=\|v\|_{L^{2}\left(\mathbb{R}^{n}\right)}^{2} & =h^{n} \int_{\mathbb{R}^{n / \Gamma}} \| v\left(h y+h(\cdot) \|_{\ell^{2}(\Gamma)}^{2} d y\right. \\
& =h^{n} \int_{\mathbb{R}^{n} / \Gamma}\left\|u_{y}\right\|_{\tau_{h y} V_{0}}^{2} d y .
\end{aligned}
$$

If we take now $\chi$ as in Proposition 3.1, and consider $\chi\left(x, h D_{x}\right)$ as acting on $\mathscr{S}^{\prime}\left(\mathbb{R}_{x}^{n} \times \mathbb{R}_{y}^{n}\right)$ with $y$ as a parameter, we see by (3.6) that the analog of Proposition 3.1 holds for $L_{0}$. Namely:

$$
\|u\|_{L_{0}}^{2}=h^{n} C_{n}\left(\chi^{w}\left(x, h D_{x}\right) u, u\right)_{L^{2}\left(\mathbb{R}_{x}^{n} \times \mathbb{R}_{y}^{n} / \Gamma\right)}
$$

We will now prove the analog of Proposition 3.2 for $L_{0}$. Let $T_{\gamma^{*}}$ be the operator multiplication by $e^{i((x / h)-y) \gamma^{*}}$ and notice that $T_{\gamma^{*}} u=u$ for $u$ in $L_{0}$ and $\gamma^{*} \in \Gamma^{*}$.

Proposition 3.6. For any $u \in L_{0}$, there exists $u_{0} \in L^{2}\left(\mathbb{R}_{x}^{n} \times \mathbb{R}_{y}^{n /} \Gamma\right)$ such that:

The property (3.2) holds for $A=A(x, \xi)$ (independent of $y, \eta$ ) if we replace $L^{2}\left(\mathbb{R}^{n}\right)$ by $L^{2}\left(\mathbb{R}_{x}^{n} \times \mathbb{R}_{y}^{n} / \Gamma\right)$.

The constants $C_{N}$ are $\mathcal{O}\left(\|u\|_{L_{0}}\right)$.

$$
\begin{aligned}
& \text { If } u_{\gamma^{*}}=T_{\gamma^{*}} u_{0} \text { then: } u=\sum_{\gamma^{*} \in \Gamma^{*}} u_{\gamma^{*}}, \\
& \text { where the series converges in } S^{\prime}\left(\mathbb{R}_{x}^{n}, K_{0}\right) \text {. }
\end{aligned}
$$

Conversely if $u_{0} \in L^{2}\left(\mathbb{R}_{x}^{n} \times \mathbb{R}_{y}^{n} / \Gamma\right)$ satisfies (3.8) and if $u_{\gamma^{*}}=T_{\gamma^{*}} u_{0}$, then the series $\sum_{\gamma^{*} \in \Gamma^{*}} u_{\gamma^{*}}$ converges in $\mathscr{S}^{\prime}\left(\mathbb{R}_{x}^{n}, K_{0}\right)$ towards a distribution $u$ in $L_{0}$ with $\|u\|_{L_{0}}$ bounded by a constant times $h^{n / 2}$ times the sum of $\left\|u_{0}\right\|_{L^{2}\left(\mathbb{R}_{x}^{n} \times \mathbb{R}_{y}^{n} / \Gamma\right)}$ and a finite number of the $C_{N}$ in (3.8).

The proof is similar to that of Proposition 3.2 and we omit it.

Proposition 3.6 gives a proof of the fact that $L_{0} \subset \mathscr{S}^{\prime}\left(\mathbb{R}_{x}^{n}, K_{0}\right)$. A more direct proof can be obtained by computing the scalar product of $u=\sum_{\gamma \in \Gamma} v(x) \delta(x-h(y-\gamma))$ with $\varphi \in \mathscr{S}\left(\mathbb{R}_{x}^{n} \times \mathbb{R}_{y}^{n} / \Gamma\right)$ and by estimating it by $\|v\|_{L^{2}\left(\mathbb{R}^{n}\right)}$ times a seminorm of $\varphi$ in $S\left(\mathbb{R}_{x}^{n}, L^{2}\left(\mathbb{R}_{y}^{n} / \Gamma\right)\right)$.

The same argument gives also that

$$
L_{m}=\left\{u \in L_{0}\left|\Lambda_{\alpha}^{w}\left(h D_{x}+A(x)\right) u \in L_{0},\right| \alpha \mid \leqq m\right\},
$$

where $\Lambda_{\alpha}(\xi)$ is the operator valued symbol $\left(\left(\xi+D_{y}\right)^{\alpha}\right)^{w}$.

One also gets that:

$$
L_{m} \subset \mathscr{S}^{\prime}\left(\mathbb{R}_{x}^{n}, H^{m}\left(\mathbb{R}_{y}^{n} / \Gamma\right)\right)
$$


We can now state prove the main results of this section, namely the spectral reduction of $P_{0}$. We keep the notations of Sect. II:

Theorem 3.7. Assume (H1) to (H5). Then, for $h>0$ small enough and $\lambda \in \mathscr{V}, \mathscr{P}^{w}\left(x, h D_{x}+A(x), \lambda\right)$ is uniformly bounded from $L_{m} \oplus\left(V_{0}\right)^{N}$ to $L_{0} \oplus\left(V_{0}\right)^{N}$, and has the uniformly bounded two sided inverse $\mathscr{E}^{w}\left(x, h D_{x}+A(x), \lambda, h\right)$ from $L_{0} \oplus\left(V_{0}\right)^{N}$ to $L_{m} \oplus\left(V_{0}\right)^{N}$.

Proof. We already know that $\mathscr{P}^{w}\left(x, h D_{x}+A(x), \lambda\right): \mathscr{S}^{\prime}\left(\mathbb{R}^{n} ; H^{m}\left(\mathbb{R}^{n} / \Gamma\right)\right) \times \mathscr{S}^{\prime}\left(\mathbb{R}^{n}\right)^{N} \rightarrow$ $\mathscr{S}^{\prime}\left(\mathbb{R}^{n} ; L^{2}\left(\mathbb{R}^{n} / \Gamma\right)\right) \times \mathscr{S}^{\prime}\left(\mathbb{R}^{n}\right)^{N}$ is bijective with inverse given by $\mathscr{E}^{w}\left(x, h D_{x}+A(x), \lambda ; h\right)$. So, we only have to show that $\mathscr{P}^{w}\left(x, h D_{x}+A(x)\right)$ and $\mathscr{E}^{w}\left(x, h D_{x}+A(x)\right)$ are bounded between $L_{m} \oplus V_{0}^{N}$ and $L_{0} \oplus V_{0}^{N}$ as indicated.

We already know that $P^{w}\left(x, h D_{x}+A(x)+D_{y}\right)$ is uniformly bounded from $L_{m}$ to $L_{0}$. It then remains to show that the following operators are uniformly bounded:

$$
\begin{array}{r}
R_{+}^{w}\left(x, h D_{x}+A(x)\right): L_{0} \rightarrow V_{0}^{N}, \\
R_{-}^{w}\left(x, h D_{x}+A(x)\right): V_{0}^{N} \rightarrow L_{m}, \\
E_{+}^{w}\left(h D_{x}+A(x), \lambda ; h\right): V_{0}^{N} \rightarrow L_{m}, \\
E_{-}^{w}\left(x, h D_{x}+A(x), \lambda ; h\right): L_{0} \rightarrow V_{0}^{N}, \\
E^{w}\left(x, h D_{x}+A(x), \lambda ; h\right): L_{0} \rightarrow L_{m}, \\
E_{-+}^{w}\left(x, h D_{x}+A(x), \lambda ; h\right): V_{0}^{N} \rightarrow V_{0}^{N} .
\end{array}
$$

Here (3.16) is a consequence of Proposition 3.3, and (3.11)-(3.15) can all be proved essentially as in the proof of this proposition. Let us show for instance (3.13). It suffices to show that for all $\alpha \in \mathbb{N}^{n}$ with $|\alpha| \leqq m, \Lambda_{\alpha}^{w}\left(h D_{x}+A(x)\right) \circ E_{+}^{w}\left(x, h D_{x}+A(x)\right)$ is uniformly bounded from $V_{0}^{N}$ to $L_{0}$. This composed operator is of the form $C^{w}\left(x, h D_{x}+A(x), \lambda ; h\right)$ with $C(x, \xi, \lambda ; h)$ in $S^{0}\left(\mathbb{R}^{2 n} ; \mathscr{L}\left(\mathbb{C}^{N} ; L^{2}\left(\mathbb{R}^{n} / \Gamma\right)\right)\right)$, and further:

$$
T_{\gamma^{*}} C^{w}\left(x, h D_{x}+A(x)\right)=C^{w}\left(x, h D_{x}+A(x)\right) \tau_{\gamma^{*}}
$$

for all $\gamma^{*} \in \Gamma^{*}$ (cf. (2.11) with $\mathscr{P}$ replaced by $\mathscr{E}$ ).

Let $u \in V_{0}^{N}$, and decompose $u=\sum_{\gamma^{*}} u_{\gamma^{*}}$ as in Proposition 3.2. Then we have $C^{w}\left(x, h D_{x}+A(x)\right) u=\sum v_{\gamma^{*}}$ with

$$
v_{\gamma^{*}}=C^{w}\left(x, h D_{x}+A(x)\right) u_{\gamma^{*}}=T_{\gamma^{*}} C^{w}\left(x, h D_{x}+A(x)\right) u_{0},
$$

and thus we are in a situation where Proposition 3.6 applies and gives the result.

Corollary 3.8. For $\lambda \in \mathscr{V}$ and $h>0$ sufficiently small, we have: $\lambda \in \sigma\left(P^{w}\left(h y, y, D_{y}+\right.\right.$ $A(h y))$ ) (where the operator is equipped with the domain $H_{m, A}$ ) if and only if $0 \in \sigma\left(E_{-+}^{w}\left(x, h D_{x}+A(x), \lambda ; h\right)\right)$, where this last operator is considered as a bounded operator: $V_{0}^{N} \rightarrow V_{0}^{N}$.

Proof. This follows from the last theorem and the two formulas:

$$
\begin{aligned}
\left(P^{w}-\lambda\right)^{-1} & =E^{w}-E_{+}^{w}(\lambda)\left(E_{-+}^{w}(\lambda)\right)^{-1} E_{-}^{w}(\lambda), \\
\left(E_{-+}^{w}(\lambda)\right)^{-1} & =-R_{+}^{w}\left(P^{w}-\lambda\right)^{-1} R_{-}^{w},
\end{aligned}
$$

where all these operators have to be considered as bounded ones on the spaces given by Theorem 3.7. 
Remark 3.9. Using the same idea as in Remark 3.4, one can also conjugate $E_{-+}^{w}\left(x, h D_{x}+A(x), \lambda, h\right)$ by the semiclassical Fourier transform

$$
F u(\xi, h)=(2 \pi h)^{-n / 2} \int e^{-i y \cdot \xi / h} u(y) d y,
$$

henceforth replacing

by

$$
E_{-+}^{w}\left(x, h D_{x}+A(x), \lambda, h\right)
$$

$$
E_{-+}^{w}\left(h D_{\xi}, \xi+A\left(h D_{\xi}\right), \lambda, h\right),
$$

(see [Hö]) and $\left(V_{0}\right)^{N}$ by $L^{2}\left(\mathbb{R}^{n} / \Gamma^{*}, \mathbb{C}^{N}\right)$, where $\mathbb{R}^{n} / \Gamma^{*}$ is given with its manifold structure. In this form it might be more transparent to derive Bohr-Sommerfeld type quantization rules to decide when $0 \in \sigma\left(E_{-+}^{w}\left(x, h D_{x}+A(x), \lambda, h\right)\right.$. We remark that the Stark ladders of resonances for a periodic crystal in a constant electric field (which is outside the scope of this work) are obtained precisely in this way.

It is of some interest to see what kind of Grushin problem we obtain for the original operator if we compose the Grushin operator of Theorem 3.7 with the earlier identifications. We recall that we have the unitary map $L^{2}\left(\mathbb{R}^{n}\right) \ni u \mapsto$ $f=v(x) \sum_{\gamma \in \Gamma} \delta(x-h(y+\gamma)) \in L_{0}$, defined by $v(h y)=u(y)$. We shall "compute" $R_{+}^{w}\left(x, h D_{x}+A(x)\right) f$ in terms of $u$. In order to simplify the notations we shall assume that $N=1$ and write $\varphi=\varphi_{1}, \psi=\psi_{1}$. (Equivalently, if we do not assume $N=1$, we shall compute the $j$-component of $R_{+}^{w}\left(x, h D_{x}+A(x)\right) f$ for some fixed $\left.j\right)$. Recall that $\varphi(x, \xi ; y)=\psi(x, \xi ; y) e^{-i y \cdot \xi}$, that $\psi$ is $\Gamma^{*}$-periodic in $\xi$ and $(\Gamma, \xi)$-Floquet periodic in $y$. We also recall that the Weyl symbol $R_{+}(x, \xi)$ is given by

$$
R_{+}(x, \xi) u=\int_{\mathbb{R}^{n / \Gamma}} u(y) \overline{\varphi(x, \xi ; y)} d y .
$$

Hence if $E$ is a fundamental domain of $\Gamma$ :

$$
\begin{aligned}
& R_{+}^{w}\left(x, h D_{x}+A(x)\right) f(x) \\
& =(2 \pi h)^{-n} \iint e^{i(x-\tilde{x}) \xi / h} R_{+}\left(\frac{x+\tilde{x}}{2}, \xi+A\left(\frac{x+\tilde{x}}{2}\right)\right) f(\tilde{x}, \cdot) d \tilde{x} d \xi \\
& =(2 \pi h)^{-n} \iint e^{i(x-\tilde{x}) \xi / h} \int_{E} f(\tilde{x}, y) \overline{\left(\frac{x+\tilde{x}}{2}, \xi+A\left(\frac{x+\tilde{x}}{2}\right) ; y\right)} d y d \tilde{x} d \xi \\
& =(2 \pi h)^{-n} \iint^{i(x-\tilde{x}) \xi / h} v(\tilde{x}) \sum_{\gamma \in \Gamma} \int_{E} \delta(\tilde{x}-h \gamma-h y) \overline{\left(\frac{x+\tilde{x}}{2}, \xi+A\left(\frac{x+\tilde{x}}{2}\right) ; y\right)} d y d \tilde{x} d \xi \text {. }
\end{aligned}
$$

If $\gamma=\gamma(\tilde{x}) \in \Gamma$ is the unique element with $y=\frac{\tilde{x}}{\operatorname{def}} \frac{\tilde{h}}{h}-\gamma \in E$, we see that the sum in the last integral reduces to

$$
\varphi\left(\frac{x+\tilde{x}}{2}, \xi+A\left(\frac{x+\tilde{x}}{2}\right) ; \frac{\tilde{x}}{h}-\gamma(\tilde{x})\right) .
$$

Using then the $\Gamma$-periodicity in $y$ of $\varphi$, the sum reduces to

$$
\overline{\varphi\left(\frac{x+\tilde{x}}{2}, \xi+A\left(\frac{x+\tilde{x}}{2}\right) ; \frac{\tilde{x}}{h}\right)} .
$$




\section{Hence}

$$
R_{+}^{w}\left(x, h D_{x}+A(x)\right) f(x)=(2 \pi h)^{-n} \iint e^{i(x-\tilde{x}) \xi / h} \overline{\left(\frac{x+\tilde{x}}{2}, \xi+A\left(\frac{x+\tilde{x}}{2}\right) ; \frac{\tilde{x}}{h}\right)} v(\tilde{x}) d \tilde{x} d \xi
$$

Here we recall that $v(\tilde{x})=u\left(\frac{\tilde{x}}{h}\right)$ so if we make the change of variables $\tilde{x}=h \tilde{y}$ and the substitution $x=h y$, we get

$$
\begin{aligned}
\left(R_{+}^{w}\left(x, h D_{x}+A(x)\right) f\right)(h y) & =(2 \pi)^{-n} \iint e^{i(y-\tilde{y}) \eta} \overline{\varphi\left(h \frac{y+\tilde{y}}{2}, \eta+A\left(h \frac{y+\tilde{y}}{2}\right) ; \tilde{y}\right)} u(\tilde{y}) d \tilde{y} d \eta \\
& \underset{\text { def }}{=}\left(\tilde{R}_{+} u\right)(y) .
\end{aligned}
$$

The distribution kernel of $\tilde{R}_{+}$becomes:

$$
\begin{aligned}
& K(y, \tilde{y})=(2 \pi)^{-n} \int e^{i(y-\tilde{y}) \eta} \overline{\left(h \frac{y+\tilde{y}}{2}, \eta+A\left(h \frac{y+\tilde{y}}{2}\right) ; \tilde{y}\right)} d \eta \\
& =(2 \pi)^{-n} \int e^{i(y-\tilde{y}) \cdot \eta+i \tilde{y}(\eta+A(h(y+\tilde{y}) / 2))} \psi\left(h \frac{y+\tilde{y}}{2}, \eta+A\left(h \frac{y+\tilde{y}}{2}\right) ; \tilde{y}\right) d \eta \\
& =e^{i \tilde{y} \cdot A(h(y+\tilde{y}) / 2)}(2 \pi)^{-n} \int e^{i y \cdot \eta} \overline{\left(h \frac{y+\tilde{y}}{2}, \eta+A\left(h \frac{y+\tilde{y}}{2}\right) ; \tilde{y}\right)} d \eta \\
& =e^{i(\tilde{y}-y) A(h(y+\tilde{y}) / 2)}(2 \pi)^{-n} \int e^{i y \cdot \eta} \psi\left(h \frac{y+\tilde{y}}{2}, \eta ; \tilde{y}\right) d \eta .
\end{aligned}
$$

In general, if $f(\eta)$ is a $\Gamma^{*}$-periodic function, then

$$
\frac{1}{(2 \pi)^{n}} \int_{\mathbb{R}^{n}} e^{i y \cdot \eta} f(\eta) d \eta=\sum a_{\gamma} \delta(y-\gamma) \quad \text { with } \quad a_{\gamma}=\frac{1}{\operatorname{Vol} E^{*}} \int_{E^{*}} e^{i \gamma \cdot \eta} f(\eta) d \eta .
$$

Since $\psi(x, \eta ; \tilde{y})$ is $\Gamma$-periodic in $\eta$, we get

$$
\begin{aligned}
& K(y, \tilde{y})=\sum_{\gamma} e^{i(\tilde{y}-y) A(h(y+\tilde{y}) / 2)} \frac{1}{\operatorname{Vol} E^{*}} \int_{E^{*}} \overline{e^{-i \gamma \cdot \eta}} \overline{\psi\left(h \frac{y+\tilde{y}}{2}, \eta ; \tilde{y}\right)} d \eta \cdot \delta(y-\gamma) \\
& =\sum_{\gamma} e^{i(\tilde{y}-y) A(h(y+\tilde{y}) / 2)} \frac{1}{\operatorname{Vol} E^{*}} \int_{E^{*}} \overline{\psi\left(h \frac{y+\tilde{y}}{2}, \eta ; \tilde{y}-\gamma\right)} d \eta \cdot \delta(y-\gamma) \\
& =\sum_{\gamma} \overline{W_{A, h}\left(h \frac{\gamma+\tilde{y}}{2}, \tilde{y}-\gamma\right)} \delta(y-\gamma)
\end{aligned}
$$

with

$$
\begin{aligned}
W_{A, h}(x, y) & =e^{-i y \cdot A(x)} W(x, y), \\
W(x, y) & =\frac{1}{\operatorname{Vol}\left(E^{*}\right)} \int_{E^{*}} \psi(x, \eta ; y) d \eta .
\end{aligned}
$$


In the situation studied in $[\mathrm{He}-\mathrm{Sj} 1]$ we can take $\psi$ independent of $x$ and then $W(y)$ is a Wannier type function used in that work as well as in [Ne].

We get $\widetilde{R}_{+} u(y)=\sum \hat{R}_{+} u(\gamma) \delta(y-\gamma)$, where $\hat{R}_{+} u \in \ell^{2}(\Gamma)$ is given by:

$$
\hat{R}_{+} u(\gamma)=\int u(\tilde{y}) \overline{W_{A, h}\left(h \frac{\gamma+\tilde{y}}{2}, \tilde{y}-\gamma\right)} d \tilde{y} .
$$

In the situation of $[\mathrm{He}-\mathrm{Sj} 1]$ we start with a constant magnetic field $\frac{h}{2} \sum \sum b_{j, k} d x_{j} \wedge d x_{k}$ with $b_{j, k}=-b_{k, j}$, where contrary to [He-Sj1] we do not assume that $\left|b_{j, k}\right|$ are small. (In that paper $h=1$ at this stage.) We can then take $A x=-\frac{1}{2} B x$, and a simple computation shows that

$$
W_{A, h}\left(h \frac{\gamma+\tilde{y}}{2}, \tilde{y}-\gamma\right)=\left(T_{\gamma}^{h B} W\right)(\tilde{y})
$$

where $T_{\gamma}^{h B}$ is the magnetic translation defined in [He-Sj1]. This means that $\hat{R}_{+}$ is the same operator as $R_{+}^{h B}$ in the terminology of [He- $\left.\mathrm{Sj} 1\right]$.

Since the various identifications in our computation are unitary and since $R_{-}^{w}\left(x, h D_{x}+A(x)\right)=R_{+}^{w}\left(x, h D_{x}+A\right)^{*}$, it is clear that this operator is naturally identified with $\hat{R}_{-}=\hat{R}_{+}^{*}$. Summing up we have proved:

Corollary 3.10. Define $\hat{R}_{-}^{*}=\hat{R}_{+}$by (3.17) or rather the natural generalization of this relation for arbitrary $N$. Thus for $\lambda$ in a neighbourhood of $\lambda_{0}$, the operator

$$
\left(\begin{array}{cc}
P^{w}\left(h y, y, D_{y}+A(h y)\right)-\lambda & \hat{R}_{-} \\
\hat{R}_{+} & 0
\end{array}\right): H_{m, A} \times \ell^{2}\left(\Gamma ; \mathbb{C}^{N}\right) \rightarrow L^{2} \times \ell^{2}\left(\Gamma ; \mathbb{C}^{N}\right)
$$

is bijective with bounded inverse $\left(\begin{array}{cc}\hat{E} & \hat{E}_{+} \\ \hat{E}_{-} & \hat{E}_{-+}\end{array}\right)$. The matrix of $\hat{E}_{-+}$is equal to the matrix of $E^{w}\left(x, h D_{x}+A, \lambda ; h\right)$ acting on $V_{0}^{N}$, if we identify the latter space with $\ell^{2}\left(\Gamma ; \mathbb{C}^{N}\right)$ in the natural way.

\section{The Case of the Schrödinger Operator}

In this section, we assume that $A(x)$ is a linear function of $x$ (corresponding to the case of a constant magnetic field). Let $V(x, y) \in C^{\infty}\left(\mathbb{R}^{2 n}\right)$ be $\Gamma$-periodic with respect to $y$ and satisfy

$$
\left|\partial_{x}^{\alpha} \partial_{y}^{\beta} V(x, y)\right| \leqq C_{\alpha, \beta},
$$

on $\mathbb{R}^{2 n}$ for every $(\alpha, \beta) \in \mathbb{N}^{2 n}$. We are then interested in the operator

$$
\sum\left(D_{y_{j}}+h A_{j}(y)\right)^{2}+V(h y, y)=P^{w}\left(h y, y, D_{y}+A(h y)\right),
$$

where

$$
P(x, y, \eta)=\eta^{2}+V(x, y) .
$$

Following the procedure of Sect. II, we fix an energy level $z_{0}$, and we choose $\psi_{1}(x, \xi ; y), \ldots, \psi_{N}(x, \xi ; y)$ smooth in all variables, $\Gamma^{*}$-periodic in $\xi$ and with 
$\psi_{j}(x, \xi ; y+\gamma)=e^{i \gamma \cdot \xi} \psi_{j}(x, \xi ; y)$, such that the problem:

$$
\left(\begin{array}{cc}
\sum D_{y_{j}}^{2}+V(x, y)-z & R_{-}^{0}(x, \xi) \\
R_{+}^{0}(x, \xi) & 0
\end{array}\right): \mathscr{F}_{2, \xi} \times \mathbb{C}^{N} \rightarrow \mathscr{F}_{0} \times \mathbb{C}^{N}
$$

is bijective for $\xi \in \mathbb{R}^{n *}$, and $z$ close to $z_{0}$. Here the spaces $\mathscr{F}_{k, \xi}$ are defined in Sect. II and $R_{-}^{0}=R_{+}^{0 *},\left(R_{+}^{0}(x, \xi) u\right)_{j}=\left(u \mid \psi_{j}\right)$. Let $E_{0-+}(x, \xi, z)$ be the $N \times N$ matrix which appears in the lower right corner of the inverse of (4.4). Putting $P(x, \xi)=P^{w}\left(x, y, \xi+D_{y}\right)$, $R_{-}(x, \xi)=e^{-i y \cdot \xi} R_{-}^{0}(x, \xi), R_{+}(x, \xi)=R_{+}^{0}(x, \xi) e^{i y \cdot \xi}, \mathscr{P}(x, \xi, z)=\left(\begin{array}{cc}P(x, \xi)-z & R_{-}(x, \xi) \\ R_{+}(x, \xi) & 0\end{array}\right)$, we then know from Sect. II that when $h$ is small enough,

$$
\mathscr{P}^{w}\left(x, h D_{x}+A(x), z\right): K_{m} \oplus L^{2}\left(\mathbb{R}^{n} ; \mathbb{C}^{N}\right) \rightarrow L^{2}\left(\mathbb{R}^{n} \times\left(\mathbb{R}^{n} / \Gamma\right)\right) \oplus L^{2}\left(\mathbb{R}^{n} ; \mathbb{C}^{N}\right)
$$

is bijective and has the uniformly bounded inverse $\mathscr{E}^{w}\left(\dot{x}, h D_{x}+A(x), z ; h\right)$. If $E_{-+}\left(x, h D_{x}+A(x), z ; h\right)$ is the $N \times N$ block in the lower right corner, then $E_{-+}(x, \xi, z ; h) \in S^{0}$ has a complete asymptotic expansion in powers of $h$ and the leading term is $E_{0-+}(x, \xi, z)$. Moreover we know that $E_{-+}$is $\Gamma^{*}$-periodic with respect to $\xi$. The main result of Sect. III tells us that $z \in \sigma\left(P^{w}\left(h y, y, D_{y}+A(h y)\right)\right)$ (as an operator acting on $\left.L^{2}\left(\mathbb{R}^{n}\right)\right)$ iff $0 \in \sigma\left(E_{-+}^{w}\left(x, h D_{x}+A(x), z ; h\right)\right)$ ), where $E_{-+}^{w}$ now acts on the space $V_{0}^{N}$ (of functions which are $\Gamma^{*}$-periodic in the dual variable).

We now discuss two special cases:

1. The Case of Periodic Potentials. We here assume that $V=V(y)$ is independent of $x$ (and $\Gamma$-periodic in $y$ ). If we choose $\varphi_{j}=\varphi_{j}(\xi ; y)$ independent of $x$, then the symbol $\mathscr{P}=\mathscr{P}(\xi)$ is independent of $x$. The operator $\mathscr{P}^{w}\left(h D_{x}+A(x)\right)$ then acquires some additional symmetry properties: We introduce the magnetic translation

$$
T_{v}^{A} u(x)=e^{-i A(v) \cdot x / h} u(x-v),
$$

and check that $\left[T_{v}^{A}, h D_{x_{j}}+A_{j}(x)\right]=0, \forall v \in \mathbb{R}^{n}, j=1, \ldots, n . T_{v}^{A}$ may be viewed as a $h$-Fourier integral operator associated to the affine linear map: $(y, \eta) \mapsto$ $(y+v, \eta-A(v))$, and we have for symbols $q$ of class $S^{0}$ :

$$
\left(T_{v}^{A}\right)^{-1} q^{w}\left(x, h D_{x}\right) T_{v}^{A}=q_{v}^{w}\left(x, h D_{x}\right), \quad q_{v}(x, \xi)=q(x+v, \xi-A(v)) .
$$

This remains true also for operator valued symbols and in particular $\mathscr{P}^{w}\left(h D_{x}+A(x), z\right)$ commutes with $T_{v}^{A}$, for all $v \in \mathbb{R}^{n}$. This is then also true for $\mathscr{E}^{w}\left(x, h D_{x}+A(x), z ; h\right)$ and using (4.9) we conclude that $\mathscr{E}(x, \xi ; h)$ is independent of $x$ :

$$
\mathscr{P}^{w}\left(h D_{x}+A(x), z\right)^{-1}=\mathscr{E}^{\mathfrak{w}}\left(h D_{x}+A(x), z ; h\right) .
$$

In particular for $z$ in a neighborhood of $z_{0}$ :

$$
z \in \sigma\left(\sum\left(D_{y_{j}}+A_{j}(h y)\right)^{2}+V(y)\right) \Leftrightarrow 0 \in \sigma\left(E_{-+}^{w}\left(h D_{x}+A(x), z ; h\right)\right),
$$

where $E_{-+}(\xi, z ; h)$ is $\Gamma^{*}$-periodic in $\xi$ and $E_{-+}^{w}$ acts on $V_{0}^{N}$.

We further notice in this case that the operators $P_{z}$ in Remark 1.2 have the same spectrum since

$$
\sum\left(D_{y_{j}}+A_{j}(z+h y)\right)^{2}+V(y)=e^{-i y \cdot A(z)}\left(\sum\left(D_{y_{j}}+A_{j}(h y)\right)^{2}+V(y)\right) e^{i y \cdot A(z)} .
$$

Hence $P^{w}\left(y, h D_{x}+A(x)+D_{y}\right)$ has the same spectrum as

$$
\sum\left(D_{y_{j}}+A_{j}(h y)\right)^{2}+V(y)
$$


so we may ignore Sect. III, and consider $E_{-+}^{w}\left(h D_{x}+A(x), z ; h\right)$ as a bounded operator on $L^{2}\left(\mathbb{R}^{n} ; \mathbb{C}^{N}\right)$ in the equivalence $(4.11)$. This result was obtained in [He-Sj1] by use of Wannier functions and some implicit arguments. See also Nenciu [Ne].

2. Quasiperiodic Potentials with Vanishing Magnetic Fields. We assume that $V(x, y)$ is $\Gamma$-periodic with respect to $y$ (as before) and $\tilde{\Gamma}$-periodic with respect to $x$, where $\tilde{\Gamma}$ is a second lattice. We shall not assume that $A$ vanishes right away. We can then choose $\varphi_{j}$ in (4.4) with the additional property: $\varphi_{j}(x+\tilde{\gamma}, \xi ; y)=\varphi_{j}(x, \xi ; y)$, $\forall \tilde{\gamma} \in \tilde{\Gamma}$ (trivially since we may even take $\varphi_{j}$ independent of $x$ ). This implies that $\mathscr{P}(x, \xi, z)$ appearing in $(4.5)$ is not only " $\Gamma^{*}$-periodic with respect to $\xi$ ", but also $\tilde{\Gamma}$-periodic with respect to $x$. Considering magnetic translations $T_{y}^{A}$ as in case 1 , we see that $\mathscr{P}^{w}\left(x, h D_{x}+A(x), z\right)$ commutes with all $T_{v}^{A}$ with $v \in \tilde{\Gamma}$. Then $\mathscr{E}^{w}\left(x, h D_{x}+A(x), z ; h\right)$ will have the same property and we conclude that $\mathscr{E}(x, \xi, z ; h)$ is $\tilde{\Gamma}$-periodic with respect to $x$. In particular:

$$
z \in \sigma\left(\sum\left(D_{y_{j}}+h A_{j}(y)\right)^{2}+V(h y, y)\right) \Leftrightarrow 0 \in \sigma\left(E_{-+}^{w}\left(x, h D_{x}+A(x), z ; h\right)\right),
$$

where $E_{-+}^{w}$ acts on $V_{0}^{N}$ and $E_{-+}(x, \xi ; h)$ is $\tilde{\Gamma}$-periodic with respect to $x$ and $\Gamma^{*}$-periodic with respect to $\xi$.

If

$$
\tilde{\Gamma}+h \Gamma \text { is dense in } \mathbb{R}^{n} \text {, }
$$

then we can let $E_{-+}^{w}\left(x, h D_{x}+A(x), z ; h\right)$ operate on $L^{2}\left(\mathbb{R}^{n} ; \mathbb{C}^{N}\right)$, the reason being again that for every $x \in \mathbb{R}^{n}$, the operator

$$
P_{x}=P^{w}\left(x+h y, y ; D_{y}+A(x+h y)\right)=P^{w}\left(x+h y, y ; D_{y}+h A(y)+A(x)\right)
$$

is isospectral to $P_{0}$.

In fact, conjugating $P_{x}$ with $e^{-i A(x) \cdot y}$, we see that $P_{x}$ is unitarily equivalent to:

$$
P^{w}\left(x+h y, y, D_{y}+h A(y)\right) \text {. }
$$

Let $\tilde{T}_{v}^{A}$ be defined as $T_{v}^{A}$ but with $h$ replaced by $1 / h$. Then $\tilde{T}_{v}^{A}$ commutes with $\left(D_{y}+h A(y)\right)^{w}$. Conjugating (4.14) with $\widetilde{T}_{\gamma}^{A}, \gamma \in \Gamma$ and using also that $P(x, y ; \eta)$ is $\tilde{\Gamma}$-periodic in $x$ and $\Gamma$-periodic in $y$, we see that for all $\gamma \in \Gamma, \tilde{\gamma} \in \tilde{\Gamma}$, the operator (4.14) is unitarily equivalent to:

$$
P^{w}\left(x+h \gamma+\tilde{\gamma}+h y, y ; D_{y}+h A(y)\right) .
$$

Using the assumption (4.13) we can take a sequence $\left(\gamma_{j}, \tilde{\gamma}_{j}\right) \in \Gamma \times \tilde{\Gamma}, j=1,2, \ldots$ such that $x+h \gamma_{j}+\tilde{\gamma}_{j} \rightarrow 0$. Then $V\left(x+h \gamma_{j}+\tilde{\gamma}_{j}, y\right) \rightarrow V(0, y)$ uniformly and we conclude that $P_{x}$ is isospectral to $P_{0}$, as stated.

We now take $n=1, A=0$, and we shall see that we can get an effective hamiltonian which is close to Harper's operator $\cos \left(h D_{x}\right)+\cos x$. We take $V_{\lambda}(x, y)=$ $U_{\lambda}(x)+\lambda^{2} W(y)$, where $\lambda \geqq 1$ is a large parameter. We also let $\Gamma=\mathbb{Z}, \tilde{\Gamma}=\Gamma^{*}=2 \pi \mathbb{Z}$. We assume that $W(y) \geqq 0$ with equality precisely on $\mathbb{Z}$, and also that $W^{\prime \prime}(0)>0$. We first consider

$$
D_{y}^{2}+\hat{\lambda}^{2} W(y)=\lambda^{2}\left(\left(\tilde{h} D_{y}\right)^{2}+W(y)\right), \quad \tilde{h}=\frac{1}{\lambda} .
$$

We can here apply known results concerning the tunnel effect in periodic semi- 
classical situations of Harrell [Ha], Outassourt [OH], Simon [Si], and we know that the first band in the spectrum of (4.16) is of the form

$$
\left[\lambda E(\lambda)-\lambda^{5 / 2} a(\lambda) e^{-S_{0} \lambda}, \lambda E(\lambda)+\lambda^{5 / 2} a(\lambda) e^{-S_{0} \lambda}\right],
$$

where $S_{0}>0$ is a certain complex action between neighboring wells, and $E(\lambda), a(\lambda) \in S_{1,0}^{0}\left(\mathbb{R}_{+}\right)$are real valued classical symbols of order 0 with asymptotic expansions:

$$
E(\lambda) \sim E_{0}+E_{1} \lambda^{-1}+\cdots, \quad a(\lambda) \sim a_{0}+a_{1} \lambda^{-1}+\cdots,
$$

with $E_{0}, a_{0}>0$. Moreover, for $\lambda>0$ large enough, the band (4.17) is all of the spectrum of (4.16) in the half axis $\left.]-\infty, 2 \lambda E_{0}\right]$.

The band (4.17) is generated by the Floquet eigenvalue:

$$
\mu_{\lambda}(\xi)=\lambda E(\lambda)+\lambda^{5 / 2} a(\lambda) e^{-S_{0} \lambda}(\cos \xi+r(\lambda, \xi)),
$$

where

$$
\left|\partial_{\lambda}^{k} \partial_{\xi}^{\ell} r(\lambda, \xi)\right| \leqq C_{k, \ell} e^{-(1 / 2) S_{0} \lambda}
$$

for all $k, \ell$.

We take

$$
U=U_{\lambda}(x)=\mu_{\lambda}(x)-2 \lambda E(\lambda) .
$$

Choosing $\mathscr{V}$ to be a complex neighborhood of the interval (4.17), we can take $N=1$ in our choice of Grushin problem with $\varphi_{1}$ independent of $x$, $\left(D_{y}^{2}+\lambda^{2} W\right) \varphi_{1}=\mu_{\lambda}(\xi) \varphi_{1}$, and choose this problem in such a way that

$$
E_{0-+}(x, \xi, z)=z-\lambda^{5 / 2} a(\lambda) e^{-S_{0} \lambda}(\cos \xi+r(\lambda, \xi)+\cos x+r(\lambda, x)) .
$$

We are therefore quite close to Harper's operator. It is quite likely that (4.20) can be extended to a large band around the real axis and that we get a corresponding result for $E_{-+}(x, \xi, z ; h)$. In order to apply the results of [He-Sj3], one would also need Fourier invariance: $E_{-+}(x, \xi, z ; h)=E_{-+}(\xi,-x, z ; h)$ (and another simpler property which may be less important). Perhaps this is also possible to obtain by means of some delicate correction terms depending both on $x$ and $y$ in the choice of $V_{\lambda}(x, y)$. A more natural solution would be however to extend the study in $[\mathrm{He} \sim \mathrm{Sj} 3]$ to the case without Fourier invariance.

\section{Appendix A. Magnetic Differential Operators and Sobolev Spaces}

Here, we shall discuss magnetic Sobolev spaces, and we assume (H.5). We define for $m \in \mathbb{N}$ :

$$
H_{A}^{m}=\left\{u \in L^{2}\left(\mathbb{R}^{n}\right) ;\left(D_{x}+A(x)\right)^{\alpha} u \in L^{2}\left(\mathbb{R}^{n}\right),|\alpha| \leqq m\right\} .
$$

Using the composition formula for Weyl quantizations, and the fact that for any $\alpha \in \mathbb{N}^{n}, \beta \in \mathbb{N}^{2 n},|\beta| \geqq 1$, we have:

$$
D_{x, \xi}^{\beta}\left((\xi+A(x))^{\alpha}\right)=\sum_{\gamma<\alpha} a_{\alpha \beta \gamma}(x)(\xi+A(x))^{\gamma},
$$

where the $a_{\alpha \beta \gamma}$ 's and all their derivatives are bounded functions on $\mathbb{R}^{n}$, it is not difficult to see (by induction on $|\alpha|$ ) that for any $\alpha \in \mathbb{N}^{n}$ and any function $a(x)$, 
bounded with all its derivatives:

$$
a(x)\left(D_{x}+A(x)\right)^{\alpha}=\left[a(x)\left(D_{x}+A(x)\right)^{\alpha}\right]^{w}+\sum_{\beta<\alpha}\left[b_{\alpha \beta}(x)\left(D_{x}+A(x)\right)^{\beta}\right]^{w},
$$

where the $b_{\alpha \beta}$ 's and all their derivatives are bounded on $\mathbb{R}^{n}$.

We can also deduce from (A.1) that:

$$
\left[a(x)\left(D_{x}+A(x)\right)^{\alpha}\right]^{w}=a(x)\left(D_{x}+A(x)\right)^{\alpha}+\sum_{\beta<\alpha} c_{\alpha \beta}(x)\left(D_{x}+A(x)\right)^{\beta},
$$

where the $c_{\alpha \beta}$ 's have the same property as the $b_{\alpha \beta}$ 's.

In view of (A.1), (A.2), we then get the same space $H_{A}^{m}$ if we replace $\left(D_{x}+A(x)\right)^{\alpha}$ by $\left[\left(D_{x}+A(x)\right)^{\alpha}\right]^{w}$ in its definition.

Proposition A.1. $H_{A}^{m}$ is a Hilbert space in which $C_{0}^{\infty}$ is dense.

Proof. It is enough to prove the density. Let $u \in H_{A}^{m}$ and $\chi_{j} \in C_{0}^{\infty}(|x|<j+1)$ with $\chi_{j}(x)=1$ for $|x| \leqq j$ and $\left|\partial^{\alpha} \chi_{j}(x)\right| \leqq C_{\alpha}$ for all $\alpha \in \mathbb{N}^{n}$ with $C_{\alpha}$ independent of $j(j \in \mathbb{N})$. Clearly $\chi_{j} u \in H_{\text {comp }}^{m}\left(\mathbb{R}^{n}\right) \subset H_{A}^{m}$. Moreover, for $|\alpha| \leqq m$ :

$$
\left(D_{x}+A(x)\right)^{\alpha}\left(\chi_{j} u\right)=\sum_{\beta \leqq \alpha} C_{\alpha \beta}\left(D_{x}^{\beta} \chi_{j}\right)\left(D_{x}+A(x)\right)^{\alpha-\beta} u,
$$

where the $C_{\alpha \beta}$ 's are constants, and $C_{\alpha 0}=1$. Thus

$$
\left(D_{x}+A(x)\right)^{\alpha}\left(\chi_{j} u\right) \rightarrow\left(D_{x}+A(x)\right)^{\alpha} u \text { in } L^{2} \quad(\text { as } j \rightarrow+\infty) .
$$

Hence, every $u$ in $H_{A}^{m}$ can be approximated by elements with compact support, and each $u$ in $H_{A}^{m}$ with compact support can be approximated by $C_{0}^{\infty}$-functions by means of a standard regularization.

Notice that we have the inclusion:

$$
H_{\text {comp }}^{m} \subset H_{A}^{m} \subset H_{\mathrm{loc}}^{m} .
$$

We next introduce differential operators. Let

with

$$
P(x, \xi)=\sum_{|\alpha| \leqq m} a_{\alpha}(x) \xi^{\alpha}
$$

$$
\left|\partial_{x}^{\beta} a_{\alpha}(x)\right| \leqq C_{\beta} \quad(\forall \alpha, \beta, x) .
$$

Then $\left|\partial_{x}^{\alpha} \partial_{\xi}^{\beta} P(x, \xi)\right| \leqq C_{\alpha \beta}\langle\xi\rangle^{m-|\beta|}$ (where $\langle\xi\rangle=\left(1+\xi^{2}\right)^{1 / 2}$ ) for any $x, \xi$ in $\mathbb{R}^{n}$ and $\alpha, \beta$ in $\mathbb{N}^{n}$.

We then put $p_{j}(x, \xi)=\sum_{|\alpha|=j} a_{\alpha}(x) \xi^{\alpha}, p=p_{m}$, and we assume that $m$ is even and for some $C_{0}>0$ :

$$
p(x, \xi) \geqq \frac{1}{C_{0}}|\xi|^{m}, \quad x \in \mathbb{R}^{n}, \quad \xi \in \mathbb{R}^{n} .
$$

We are interested in $\operatorname{Op}_{A}(P)=P^{w}\left(x, D_{x}+A(x)\right)$, which can also be written:

$$
\mathrm{Op}_{A}(P)=\sum_{|\alpha| \leqq m} b_{\alpha}(x)\left(D_{x}+A(x)\right)^{\alpha}
$$

where the $b_{\alpha}$ 's satisfy (A.4), and $b_{\alpha}=a_{\alpha}$ when $|\alpha|=m$.

It is easy to see that $\operatorname{Op}_{A}(P)$ is bounded $H_{A}^{m+k} \rightarrow H_{A}^{k}$ for every $k \in \mathbb{N}$. 
If we fix $x_{0} \in \mathbb{R}^{n}$ and put $\xi_{0}=A\left(x_{0}\right)$, we have:

$$
e^{i x \xi_{0}} \mathrm{Op}_{A}(P) e^{-i x \xi_{0}}=\sum_{|\alpha| \leqq m} b_{\alpha}(x)\left(D_{x}+A(x)-A\left(x_{0}\right)\right)^{\alpha} .
$$

This operator is bounded: $H_{A-\xi_{0}}^{m+k} \rightarrow H_{A-\xi_{0}}^{k}$. If $\Omega$ is an open set of $\mathbb{R}^{n}$, we write:

$$
\|u\|_{H_{A}^{k}(\Omega)}^{2} \stackrel{\text { def }}{=} \sum_{|\alpha| \leqq k}\left\|\left(D_{x}+A(x)\right)^{\alpha} u\right\|_{L^{2}(\Omega)}^{2}
$$

Then, thanks to (H.5), we see that the norms $\|\cdot\|_{H_{A-\xi_{0}}^{k}\left(B\left(x_{0}, 2\right)\right)}$ and $\|\cdot\|_{H^{k}\left(B\left(x_{0}, 2\right)\right)}$ are equivalent uniformly with respect to $x_{0}$. (Here $B\left(x_{0}, 2\right)$ denotes the open ball of center $x_{0}$ and radius 2 .)

The operator (A.7) can be written:

$$
\sum_{|\alpha| \leqq m} c_{\alpha}\left(x, x_{0}\right) D_{x}^{\alpha}
$$

with $c_{\alpha}\left(x, x_{0}\right)=a_{\alpha}(x)$ for $|\alpha|=m$, and $\left|\partial_{x}^{\beta} c_{\alpha}(x)\right| \leqq C_{\beta}$ for any $\alpha, x \in B\left(x_{0}, 2\right)$, and with $C_{\beta}$ independent of $x_{0}$.

Standard a piori estimates for elliptic operators then give for any $u$ in $H_{A}^{k+m}\left(B\left(x_{0}, 2\right)\right)$ :

$$
\|u\|_{H_{A}^{k+m}\left(B\left(x_{0}, 1\right)\right)} \leqq C_{k}\left[\left\|\mathrm{Op}_{A}(P) u\right\|_{H_{A}^{k}\left(B\left(x_{0}, 2\right)\right)}+\|u\|_{L^{2}\left(B\left(x_{0}, 2\right)\right)}\right]
$$

where $C_{k}$ is independent of $x_{0}$.

If we also assume, as we shall do from now on, that $P$ is real valued, then $\mathrm{Op}_{A}(P)$ is formally selfadjoint and the classical Gårding inequality becomes:

$$
\left(\mathrm{Op}_{A}(P) u, u\right)_{L^{2}} \geqq \frac{1}{C_{0}}\|u\|_{H_{A}^{m / 2}}^{2}-C_{0}\|u\|_{L^{2}}^{2}
$$

for all $u \in \mathscr{E}^{\prime}\left(B\left(x_{0}, 2\right)\right) \cap H_{A}^{m / 2}$, and with $C_{0}$ independent of $x_{0}$.

Combining (A.9) with a simple covering argument, we get:

Proposition A.2. If $u \in L^{2}\left(\mathbb{R}^{n}\right)$ and $\mathrm{Op}_{A}(P) u \in H_{A}^{k}\left(\mathbb{R}^{n}\right)$, then $u \in H_{A}^{k+m}\left(\mathbb{R}^{n}\right)$ and we have:

$$
\|u\|_{H_{A}^{k+m}} \leqq C_{k}\left[\left\|O \mathrm{pp}_{A}(P) u\right\|_{H_{A}^{k}}+\|u\|_{L^{2}}\right]
$$

where $C_{k}$ is independent of $u$.

Using a partition of unity, we also get from (A.10) (with a new constant $C_{0}$ ):

$$
\left(\mathrm{Op}_{A}(P) u, u\right)_{L^{2}} \geqq \frac{1}{C_{0}}\|u\|_{H_{A}^{m / 2}}^{2}-C_{0}\|u\|_{L^{2}}^{2}
$$

for all $u \in H_{A}^{m}$. (The commutator terms are estimated by $C\|u\|_{H_{A}^{(m / 2)-1}} \cdot\|u\|_{H_{A}^{m / 2}}$ and we can use that $\|u\|_{H_{A}^{k-1}} \leqq \varepsilon\|u\|_{H_{A}^{k}}+C_{\varepsilon, k}\|u\|_{L^{2}}$ for any $\varepsilon>0$ ).

If we consider $\mathrm{Op}_{A}(P)$ as a symmetric operator with domain $C_{0}^{\infty}\left(\mathbb{R}^{n}\right),(\mathrm{A} .12)$ shows that $\mathrm{Op}_{A}(P)$ is semibounded from below, and admits at least one self-adjoint extension (the Friedrichs one). Proposition A.2 actually shows that $\mathrm{Op}_{A}(P)$ is essentially self-adjoint, and that the domain of its unique self-adjoint extension is $H_{A}^{m}$. 


\section{Appendix B. Pseudodifferential Operators with Operator Valued Symbols}

We review some standard facts. Our main reference is here the unpublished work of A. Balazard-Konlein [Ba].

We denote a point $(x, \xi) \in \mathbb{R}^{2 n}$ by $X$. We shall consider a family of Hilbert spaces $\mathscr{A}_{X}, X \in \mathbb{R}^{2 n}$ satisfying:

$$
\mathscr{A}_{X}=\mathscr{A}_{Y} \text { as vector spaces for all } X, Y \in \mathbb{R}^{2 n} \text {, }
$$

There exist $N_{0} \geqq 0$ and $C \geqq 0$ such that

$$
\|u\|_{\mathscr{A}_{X}} \leqq C\langle X-Y\rangle^{N_{0}}\|u\|_{\mathscr{A}_{Y}} \text { for all } u \in \mathscr{A}_{0}, X, Y \in \mathbb{R}^{2 n} \text {. }
$$

Let $\mathscr{B}_{X}, X \in \mathbb{R}^{2 n}$ be a second family with the same properties. We say that $p \in C^{\infty}\left(\mathbb{R}^{2 n} ; \mathscr{L}\left(\mathscr{A}_{0}, \mathscr{B}_{0}\right)\right)$ belongs to $S^{0}\left(\mathbb{R}^{2 n} ; \mathscr{L}\left(\mathscr{A}_{X}, \mathscr{B}_{X}\right)\right)$ if for every $\alpha \in \mathbb{N}^{2 n}$, there is a constant $C_{\alpha}$ such that

$$
\left\|\partial_{X}^{\alpha} p\right\|_{\mathscr{L}\left(\mathscr{A}_{X}, \mathscr{B}_{X}\right)} \leqq C_{\alpha}, \quad X \in \mathbb{R}^{2 n} .
$$

If $p$ depends on the additional parameter $\left.h \in] 0, h_{0}\right], h_{0}>0$, we say that $p$ belongs to $S^{0}\left(\mathbb{R}^{2 n} ; \mathscr{L}\left(\mathscr{A}_{X}, \mathscr{B}_{X}\right)\right)$ if so is the case for every fixed $h$ and if (B.3) holds with constants $C_{\alpha}$ which are independent of $h$.

Proposition B.1. Let $p \in S^{0}\left(\mathbb{R}^{2 n} ; \mathscr{L}\left(\mathscr{A}_{X}, \mathscr{B}_{X}\right)\right)$, where $\mathscr{A}_{X}, \mathscr{B}_{X}$ satisfy $(B .1),(B .2)$. Then $\mathrm{Op}_{h}(p)=p^{w}\left(x, h D_{x}\right)$ is uniformly continuous $\mathscr{S}\left(\mathbb{R}^{n} ; \mathscr{A}_{0}\right) \rightarrow \mathscr{S}\left(\mathbb{R}^{n} ; \mathscr{B}_{0}\right)$.

Proof. We neglect certain standard density arguments like approximating the symbols by symbols with compact support. In the formula

$$
\mathrm{Op}_{h}(p) u(x)=\iint e^{i(x-y) \xi / h} p\left(\frac{x+y}{2}, \xi\right) u(y) \frac{d y d \xi}{(2 \pi h)^{n}},
$$

where $u \in \mathscr{S}\left(\mathbb{R}^{n}, \mathscr{A}_{0}\right)$ we use the fact that:

$$
\left(1+|x-y|^{2}+|\xi|^{2}\right)^{-1}\left(1-\xi \cdot h D_{y}+(x-y) \cdot h D_{\xi}\right) e^{i(x-y) \xi / h}=e^{i(x-y) \xi / h}
$$

and obtain after $N$ steps:

$$
\begin{aligned}
\mathrm{Op}_{h}(p) u(x)= & (2 \pi h)^{-n} \iint e^{i(x-y) \xi / h}\left[\left(1+\xi h D_{y}-(x-y) h D_{\xi}\right) \circ\left(1+|x-y|^{2}+|\xi|^{2}\right)^{-1}\right]^{N} \\
& \cdot\left(p\left(\frac{x+y}{2}, \xi\right) u(y)\right) d y d \xi \\
= & (2 \pi h)^{-n} \iint e^{i(x-y) \xi / h} q(x, y, \xi, h) d y d \xi
\end{aligned}
$$

Here

$$
q(x, y, \xi ; h)=\mathcal{O}(1)(1+|\xi|+|x-y|)^{-N}(1+|x|+|y|+|\xi|)^{2 N_{0}}(1+|y|)^{-N} \text { in } \mathscr{B}_{0} .
$$

This implies:

$$
\begin{aligned}
q(x, y, \xi ; h) & =\mathcal{O}(1)(1+|\xi|)^{-(N / 2)+2 N_{0}}(1+|x-y|)^{-N / 2}(1+|y|)^{-N+2 N_{0}}(1+|x|)^{2 N_{0}} \\
& \leqq \mathcal{O}(1)(1+|\xi|)^{-(N / 2)+2 N_{0}}(1+|x-y|)^{-(N / 2)+2 N_{0}}(1+|y|)^{-N+4 N_{0}} \text { in } \mathscr{B}_{0},
\end{aligned}
$$

and choosing $N$ sufficiently large we see from this and (B.6) that

$$
\left\|\mathrm{Op}_{h}(p) u(x)\right\|_{\mathscr{B}_{0}}=\mathcal{O}(1)(1+|x|)^{-\tilde{N}} \text { for every } \tilde{N} \text {. }
$$

The derivatives in $x$ can be estimated similarly. 
The formal complex adjoint of $\mathrm{Op}_{h}(p)$ is $\mathrm{Op}_{h}\left(p^{*}\right)$, and using this remark we see that under the same assumptions as in Proposition B.1.

$$
\mathrm{Op}_{h}(p): \mathscr{S}^{\prime}\left(\mathbb{R}^{n} ; \mathscr{A}_{0}\right) \rightarrow \mathscr{S}^{\prime}\left(\mathbb{R}^{n} ; \mathscr{B}_{0}\right)
$$

We next review the composition. Let $\mathscr{C}_{\boldsymbol{X}}$ be a third family of Hilbert spaces which also satisfies (B.1), (B.2).

Proposition B.2. Let $p \in S^{0}\left(\mathbb{R}^{2 n} ; \mathscr{L}\left(\mathscr{B}_{X}, \mathscr{C}_{X}\right)\right), q \in S^{0}\left(\mathbb{R}^{2 n} ; \mathscr{L}\left(\mathscr{A}_{X}, \mathscr{B}_{X}\right)\right)$. Then $\operatorname{Op}_{h}(p)^{\circ}$ $\mathrm{Op}_{h}(q)=\mathrm{Op}_{h}(r)$, where $r \in S^{0}\left(\mathbb{R}^{2 n} ; \mathscr{L}\left(\mathscr{A}_{X}, \mathscr{C}_{X}\right)\right)$ is given by

$$
r=\left.\exp \left(\frac{i h}{2} \sigma\left(D_{x}, D_{\xi} ; D_{y}, D_{\eta}\right)\right)(p(x, \xi) q(y, \eta))\right|_{\substack{x=y \\ \xi=\eta}} \stackrel{\text { def }}{=} p \#_{h} q,
$$

where $\sigma$ is the usual symplectic 2-form.

We have the asymptotic formula:

$$
\left.r \sim \sum_{k=0}^{\infty} \frac{1}{k !}\left(\frac{i h}{2}\right)^{k}\left(\sigma\left(D_{x}, D_{\xi} ; D_{y}, D_{\eta}\right)^{k} p(x, \xi) q(y, \eta)\right)\right|_{\substack{x=y \\ \xi=\eta}}
$$

in the following standard sense: write $S^{k}=h^{-k} S^{0}{ }_{k}$ If $s_{j} \in S^{m_{j}}, j=0,1,2, \ldots$ with $0 \geqq m_{j} \searrow-\infty, j \rightarrow \infty$, then we write $s \sim \sum s_{j}$ if $s-\sum_{0}^{k} s_{j} \in S^{m_{k+1}}$ for every $k$.

Proof. The only slightly new fact to check (compared to the scalar case) is that (B.7) really defines a symbol of class $S^{0}$ and that we have (B.8). Write $(x, \xi)=X$, $(y, \eta)=Y$. Then $\exp \frac{i h}{2} \sigma\left(D_{X} ; D_{Y}\right)$ can be viewed as the operator of convolution with $C_{n} h^{-2 n} \exp \left(-\frac{2 i}{h} \sigma(X ; Y)\right)$, where the constant $C_{n}$ only depends on the dimension. Modulo the usual density arguments starting with the case when $p, q$ have compact support, we get:

$$
\begin{aligned}
p \#_{h} q(X) & =\left.C_{n} h^{-2 n} \exp \left(-\frac{2 i}{h} \sigma\right) *(p \otimes q)\right|_{Y=X} \\
& =C_{n} h^{-2 n} \iint e^{-(2 i / h) \sigma(\tilde{X}, \tilde{Y})} p(X-\tilde{X}) q(X-\tilde{Y}) d \tilde{X} d \tilde{Y} .
\end{aligned}
$$

Here we introduce a function $\chi \in C_{0}^{\infty}\left(\mathbb{R}^{2 n}\right)$ which is equal to 1 near 0 and we introduce $\chi(\tilde{X}) \chi(\tilde{Y})$ as a cutoff function in (B.9). We first examine the contribution for the remainder, that is for $1-\chi(\tilde{X}) \chi(\tilde{Y})$. On the support of the integrand we then have $|\tilde{X}|+|\tilde{Y}| \geqq \frac{1}{C_{0}}$ for some $C_{0}>0$. With

we have

$$
L=\frac{i}{2} \cdot \frac{\left(\nabla_{\tilde{X}} \sigma(\tilde{X}, \tilde{Y})\right) \cdot D_{\tilde{X}}+\left(\nabla_{\tilde{Y}} \sigma(\tilde{X}, \tilde{Y})\right) \cdot D_{\tilde{Y}}}{\left(\nabla_{\tilde{X}} \sigma(\tilde{X}, \tilde{Y})\right)^{2}+\left(\nabla_{\tilde{Y}} \sigma(\tilde{X}, \tilde{Y})\right)^{2}}
$$

$$
h L e^{-(2 i / h) \sigma(\tilde{X}, \tilde{Y})}=e^{-(2 i / h) \sigma(\tilde{X}, \tilde{Y})},
$$

and we also notice that

$$
\left|\nabla_{\tilde{X}} \sigma(\tilde{X}, \tilde{Y})\right|+\left|\nabla_{\tilde{Y}} \sigma(\tilde{X}, \tilde{Y})\right| \sim|\tilde{X}|+|\tilde{Y}| .
$$


The contribution to (B.9) under study, then becomes:

$$
\begin{aligned}
& h^{N-2 n} C_{n} \iint e^{-(2 i / h) \sigma(\tilde{X}, \tilde{Y})}\left({ }^{t} L\right)^{N}[(1-\chi(\tilde{X}) \chi(\tilde{Y})) p(X-\tilde{X}) q(X-\tilde{Y})] d \tilde{X} d \tilde{Y} \\
& \quad=C_{n} h^{N-2 n} \iint e^{-(2 i / h) \sigma(\tilde{X}, \tilde{Y})} t_{N}(X, \tilde{X}, \tilde{Y} ; h) d \tilde{X} d \tilde{Y}
\end{aligned}
$$

Here

$$
t_{N}=\mathcal{O}\left((1+|\tilde{X}|+|\tilde{Y}|)^{-N}\right) \text { in } \quad \mathscr{L}\left(\mathscr{B}_{X-\tilde{X}}, \mathscr{C}_{X-\tilde{X}}\right) \circ \mathscr{L}\left(\mathscr{A}_{X-\tilde{Y}}, \mathscr{B}_{X-\tilde{Y}}\right)
$$

so

$$
\left.t_{N}=\mathcal{O}(1)(1+|\tilde{X}|+|\tilde{Y}|)^{-N}\right)(1+|\tilde{X}|)^{2 N_{0}}(1+|\tilde{Y}|)^{2 N_{0}} \text { in } \mathscr{L}\left(\mathscr{A}_{X}, \mathscr{C}_{X}\right) .
$$

The contribution (B.11) in therefore $\mathcal{O}\left(h^{N}\right)$ in $\mathscr{L}\left(\mathscr{A}_{X}, \mathscr{C}_{X}\right)$ for every $N$, and the same holds for all derivatives with respect to $X$.

We then turn to the main contribution, from $\chi(\tilde{X}) \chi(\tilde{Y})$, which we write as

$$
\left.\exp \left(\frac{i h}{2} \sigma\left(D_{\tilde{X}} ; D_{\tilde{Y}}\right)\right)(u(X, \tilde{X}, \tilde{Y}))\right|_{\tilde{X}=\tilde{Y}=X}
$$

with

$$
u(X, \tilde{X}, \tilde{Y})=\chi(X-\tilde{X}) p(\tilde{X}) \chi(X-\tilde{Y}) q(\tilde{Y})
$$

Here $u$ has its support in a domain $|\tilde{X}-X|+|\tilde{Y}-X| \leqq$ const., so we can work directly in $\mathscr{L}\left(\mathscr{B}_{X}, \mathscr{C}_{X}\right) \circ \mathscr{L}\left(\mathscr{A}_{X}, \mathscr{B}_{X}\right) \subset \mathscr{L}\left(\mathscr{A}_{X}, \mathscr{C}_{X}\right)$, and analyze (B.12) by using the Fourier transform.

The $L^{2}$-boundedness can be established exactly as in the scalar case:

Proposition B.3. Assume $\mathscr{A}_{X}=\mathscr{A}_{0}, \mathscr{B}_{X}=\mathscr{B}_{0}, \forall X \in \mathbb{R}^{2 n}$. If $p \in S^{0}\left(\mathbb{R}^{2 n} ; \mathscr{L}\left(\mathscr{A}_{0}, \mathscr{B}_{0}\right)\right)$, then $\mathrm{Op}_{h}(p)$ is uniformly bounded:

$$
L^{2}\left(\mathbb{R}^{n} ; \mathscr{A}_{0}\right) \rightarrow L^{2}\left(\mathbb{R}^{n} ; \mathscr{B}_{0}\right) .
$$

This is the classical Calderon-Vaillancourt result which can be proved as usual with the help of Cotlar's lemma. We also have the following result proved in [He-Sj2] in the scalar case:

Proposition B.4. Let $\mathscr{A}_{X}=\mathscr{A}_{0}, \mathscr{B}_{X}=\mathscr{B}_{0}$, and assume that $\psi_{1}, \psi_{2}$, p belong to bounded sets of $S^{0}\left(\mathbb{R}^{2 n} ; \mathscr{L}\left(\mathscr{B}_{0}, \mathscr{B}_{0}\right)\right), \quad S^{0}\left(\mathbb{R}^{2 n} ; \mathscr{L}\left(\mathscr{A}_{0}, \mathscr{A}_{0}\right)\right), \quad S^{0}\left(\mathbb{R}^{2 n} ; \mathscr{L}\left(\mathscr{A}_{0}, \mathscr{B}_{0}\right)\right)$ respectively.

We also assume that $\operatorname{dist}\left(\operatorname{supp} \psi_{1}, \operatorname{supp} \psi_{2}\right) \geqq \varepsilon_{0}>0$ for some fixed $\varepsilon_{0}$. Then for every $N \in \mathbb{N}$ there exists a constant $C_{N}$, such that

$$
\left\|\mathrm{Op}_{h}\left(\psi_{1}\right) \mathrm{Op}_{h}(p) O \mathrm{Op}_{h}\left(\psi_{2}\right)\right\| \leqq C_{N} h^{N} \operatorname{dist}\left(\operatorname{supp} \psi_{1}, \operatorname{supp} \psi_{2}\right)^{-N} \text {. }
$$

\section{References}

[Ba] Balazard-Konlein, A.: Calcul fonctionnel pour des opérateurs $h$-admissibles à symbole opérateur et applications. Thèse 3ème cycle, Nantes 1985

[Be] Beals, R.: Characterization of pseudodifferential operators and applications. Duke Math. J. 44 (1), 45-57 (1977)

[Bu] Buslaev, V. S.: Semiclassical approximation for equations with periodic coefficients. Russ. Math. Surv. 42 (6), 97-125 (1987) 
[Gu-Ra-Tr] Guillot, J. C., Ralston, J., Trubowitz, E.: Semi-classical methods in solid state physics. Commun. Math. Phys. 116, 401-415 (1988)

[Ha] Harrell, E. M.: The band structure of a one dimensional periodic system in the scaling limit. Ann. Phys. 119, 351-369 (1979)

[He-Sj] Helffer, B., Sjöstrand, J.:

[1] On diamagnetism and de Haas-Van Alphen effect. Ann. I.H.P. (physique théorique) 52 (4), 303-375 (1990)

[2] Analyse semi-classique pour l'équation de Harper. Bull. S.M.F., Mémoire $n^{\circ} 34$, T. 116, Fasc. 4 (1988)

[3] Semiclassical analysis for Harpers equation III - Cantor structure of the spectrum. Bull. S.M.F., Mémoire (to appear)

[Hö] Hörmander, L.: The Weyl calculus of pseudodifferential operators. Commun. Pure App. Math. 32, 359-443 (1979)

[Ne] Nenciu, G.: Dynamics of band electrons in electric and magnetic fields: rigorous justification of the effective Hamiltonians. Rev. Mod. Phys. 63, 91-127 (1991)

[Ou] Outassourt, A.: Analyse semi-classique pour des opérateurs de Schrödinger avec potential périodique, J. Funct. Anal. 72 (1), (1987)

[Re-Si] Reed, M., Simon, B.: Methods of modern mathematical physics, Tome IV. New York: Academic Press 1975

[Si] Simon, B.: Semiclassical analysis of low lying eigenvalues III - Width of the ground state band in strongly coupled solids. Ann. Phys. 158, 415-420 (1984)

[Sk] Skriganov, M. M.: Geometric and arithmetic methods in the spectral theory of multidimensional periodic operators. Proceedings of the Steklov Institute of Mathematics, ${ }^{\circ} 2$ (1987)

Communicated by B. Simon 